\title{
A Mean-Field Matrix-Analytic Method for Bike Sharing Systems under Markovian Environment
}

\author{
Quan-Lin Li and Rui-Na Fan \\ School of Economics and Management Sciences \\ Yanshan University, Qinhuangdao 066004, P.R. China
}

February 14, 2018

\begin{abstract}
This paper proposes a novel mean-field matrix-analytic method in the study of bike sharing systems, where the Markovian environment is constructed to well express time-inhomogeneity and asymmetry of the processes that the customers rent and return the bikes. To achieve effective computability of this mean-field method, this paper provides a unified framework through three basic steps: The first one is to deal with a major challenge encountered in setting up the mean-field block-structured equations in more general bike sharing systems. Here we provide an effective technique to establish a necessary reference system which is a time-inhomogeneous queue with block structure. The second step is to prove the asymptotic independence (or propagation of chaos) in terms of the martingale limits. Note that the asymptotic independence ensures and supports that we can construct a nonlinear QBD process such that the stationary probability of problematic stations can be computed under a unified nonlinear QBD framework. Finally, in the third step we use some numerical examples to show effectiveness and computability of this mean-field matrix-analytic method, and also give valuable observation on influence of some key parameters on system performance. We hope the methodology and results given in this paper are applicable in the study of more general large-scale bike sharing systems.
\end{abstract}

Keywords: Bike sharing system; mean-field matrix-analytic method; Markovian environment; time-inhomogeneous queue; nonlinear QBD process; probability of problematic stations. 


\section{Introduction}

In this paper, we apply the mean-field theory, combining Markov processes with timeinhomogeneous queues, to study a complicated bike sharing system with user's finite waiting rooms under a Markovian environment. To this end, we develop a novel meanfield matrix-analytic method in the study of more general bike sharing systems as follows: Setting up a block-structured system of mean-field equations by constructing a reference system: The time-inhomogeneous $\operatorname{MAP}(t) / \operatorname{MAP}(t) / 1 / \mathrm{K}+2 \mathrm{~L}+1$ queue; proving the asymptotic independence by means of the martingale limits; establishing a nonlinear QBD process such that the fixed point can be computed numerically; and using some numerical examples to give valuable observation of influence of some key parameters on system performance.

During the last decades the bike sharing systems have emerged as a public transport mode devoted to short trips, and they have widely been deployed in more than 900 major cities around the world. So far the bike sharing systems have been regarded as a promising solution to reduce traffic congestion, parking difficulties, automobile exhaust pollution, transportation noise and so on. For the history and survey papers, readers may refer to, such as, DeMaio [6], Shaheen et al. [40], Meddin and DeMaio [32, Shu et al. [42, Fishman et al. [7, Labadi et al. [18, Kaspi et al. [17] and the references therein.

For design and operations of the bike sharing systems, it has become a basic and interesting topic to assess and ensure the quality of service (abbreviated as QoS) from a user's perspective, e.g., see Kaspi et al. [17] for necessary interpretation. In general, the QoS of a bike sharing system may be evaluated from two basic points: (a) The bike non-empty. Some bikes have been parked at the stations such that any arriving customer can rent a bike from his entering station. (b) The parking non-full. Some parking places (or lockers) become empty and available so that a rider can immediately return his bike at a destination station. Based on the two points, the bike-empty or parking-full stations are called problematic stations, while the probability of problematic stations can be used to measure the QoS of the bike sharing system. In general, computing the probability of problematic stations is always very difficult and challenging. To indicate the major reason of such difficulties, from a physical point of view, Li et al. [26, 27. transformed the more general bike sharing system into a complicated closed queueing network whose customers and nodes are all virtual from the bikes, from the stations and from the roads, and provided 
an effective method to compute the stationary probability of problematic stations through deriving the product-form solution of joint stationary distribution of queue lengths. On the other hand, it is worthwhile to note that recent interesting research of bike sharing systems is also related to the probability of problematic stations. Readers may refer to, for example, the inventory management by Raviv and Kolka [36] and Schuijbroek et al. [41, optimization of the bike fleet sizes by Fricker and Gast [8], and influence of the unusable bikes by Kaspi et al. [17].

Little work has been done on numerical computation of the probability of problematic stations through applications of queueing theory and Markov processes. Important examples in the recent literature are classified as the following two aspects. (a) Simple queues: Leurent [20] used the $\mathrm{M} / \mathrm{M} / 1 / \mathrm{C}$ queue to study a vehicle-sharing system. Schuijbroek et al. [41] first evaluated the service level by means of the transient distribution of the $\mathrm{M} / \mathrm{M} / 1 / \mathrm{C}$ queue, and then established some optimal problems with respect to the vehicle routing. Raviv et al. [37] and Raviv and Kolka [36] employed the transient distribution of the $\mathrm{M}(t) / \mathrm{M}(t) / 1 / \mathrm{C}$ queue to compute the expected number of bike shortages at each station. (b) Closed queueing networks: Adelman [1] used a closed queueing network to set up an internal pricing mechanism for managing a fleet of service units, and provided a price-based policy for the vehicle redistribution. George and Xia [16] used some simple closed queueing networks to study the vehicle rental systems, and determined the optimal number of parking places in each rental location. Waserhole et al. 47] and Waserhole and Jost [46] used a simple closed queueing network, combining with the fluid approximation, to establish the Markov decision models in order to determine the optimal policy of the bike sharing system. Li et al. [26] provided a unified framework of applying the closed queueing networks whose product solution can be used to compute the stationary probability of problematic stations. Li et al. [27] further extended the modeling method of [26] to be able to study a more general bike sharing system from two key factors: Markovian arrival processes and an irreducible road graph. Under the heavy-traffic conditions, Li et al. 29] gave fluid and diffusion limits of the bike sharing system with renewal user arrivals and general riding-bike times by means of analyzing a multiclass closed queueing network.

If a bike sharing system contains $N$ stations and at most $N(N-1) / 2$ roads (or an irreducible road graph), then it can be described as a virtual closed queueing network whose analysis is complicated and difficult due to multiple virtual nodes (station or road) and many parameters in this system. See Li et al. [27, 26] for detailed interpretation. In 
this case, the mean-field theory should be one of the best approximate methods for understanding dynamic behavior of more general bike sharing systems. Note that the mean-field theory is a popular approximate method in the study of complex physical systems, while its applications contain at least two basic points: Focusing on a tagged node, and computing mean-field parameters of this tagged node through weak interactions among all the nodes. So far the available results of applying the mean-field theory to the bike sharing systems have been very limited. Fricker et al. [9] first made a pioneering seminar work for applying the mean-field theory to some heterogeneous bike sharing systems. Since then, subsequent papers have been published on this theme. Fricker and Gast [8] gave some simple mean-field models to study a space-homogeneous bike sharing system in terms of the $\mathrm{M} / \mathrm{M} / 1 / \mathrm{K}$ queue, and derived the closed-form solution both for the minimal proportion of problematic stations and for the optimal fleet sizes. Fricker and Servel [10] applied the mean-field theory to consider two-choice regulation in heterogeneous closed networks, and then dealt with a bike sharing system with multiple clusters. Fricker and Tibi [11] first studied the central limit and local limit theorems for the independent (non-identically distributed) random variables, which provide support on analysis of a generalized Jackson network with product-form solution. Then they used the limit theorems to give a stationary asymptotic analysis for the locally space-homogeneous bike sharing systems. Li et al. [23] applied the mean-field theory to discuss the bike sharing system with more random factors through a time-inhomogeneous queue and a nonlinear birth-death process, and numerically computed the fixed point which gives performance analysis of the bike sharing system.

The purpose of this paper is to improve the mean-field theory to be able to study more general bike sharing systems from two key factors: (a) A Markovian environment is constructed to well express time-inhomogeneity and asymmetry of the processes that the customers rent and return the bikes; and (b) user's finite waiting rooms are added to the stations such that the probability of problematic stations can be reduced greatly. From mathematical modeling and analysis, both the Markovian environment and the user's finite waiting rooms make analysis of the bike sharing systems more difficult and challenging. In addition, it is worthwhile to note that introduction of the Markovian environment motivates us to improve the mean-field theory to be able to set up the mean-field block-structured equations, to prove the asymptotic independence with block structure, and to establish a nonlinear Markov process which is used to compute the fixed 
point. Based on this, we develop a mean-field matrix-analytic method in the study of bike sharing systems. As a nearby research of this paper, Li and Lui [28] applied the mean-field theory to discuss a block-structured supermarket model. Li [22] provided a unified block-structured framework for the mean-field theory of stochastic big networks with weak interactions.

For the mean-field theory of stochastic networks, readers may refer to, such as, two books by Liggett [30] and Chen [3], two survey papers by Sznitman [43] and Benaim and Le Boudec 22. Since the mean-field theory was first applied to the study of largescale parallel queues (for example, supermarket models and work stealing models) by Vvedenskaya et al. [45] and Mitzenmacher [33, subsequent papers have been published on this theme, among which see Turner [44, Martin and Suhov [31, Graham [14, 15], Gast and Gaujal [12, 13, Li et al. [24, 25], Li and Lui [28, Li [22, Mukhopadhyay [34] and the references therein. On the other hand, the QBD processes often provide a useful mathematical tool for studying stochastic models such as queueing systems, manufacturing systems, communication networks and healthcare systems. Readers may refer to Chapter 3 of Neuts [35], Latouche and Ramaswami [19], Li [21] and references therein.

The main contributions of this paper are threefold. The first one is to propose a novel mean-field matrix-analytic method in the study of bike sharing systems. Note that this new method can effectively improve the descriptive and computational ability of the mean-field theory under a unified framework of nonlinear Markov processes, e.g., see Li 22 for a detailed discussion. To demonstrate such an ability by using examples, in a bike sharing system we first introduce two key factors: The Markovian environment and the user's finite waiting rooms. Then we show that the two factors may result in some major challenges when applying the mean-field theory to the bike sharing system, for example, it is always very difficult to set up a block-structured system of mean-field equations due to existence of the Markovian environment. To overcome difficulty of the block structure, Subsection 3.1 provides an effective technique for establishing a necessary reference system: A time-inhomogeneous queue $\operatorname{MAP}(t) / \operatorname{MAP}(t) / 1 / \mathrm{K}+2 \mathrm{~L}+1$. At the same time, the other key points of applying the mean-field matrix-analytic method are also discussed as follows: (i) Section 4 proves the asymptotic independence by means of the martingale limits, (ii) Section 5 establishes a nonlinear QBD process such that the fixed point can be computed numerically, and (iii) Section 6 uses the fixed point to evaluate performance measures of the bike sharing system, and specifically, to compute the stationary probability of 
problematic stations.

The second contribution of this paper is to introduce the Markovian environment, which can well express time-inhomogeneity and asymmetry of the processes that the customers rent and return the bikes in a bike sharing system. To our best knowledge, it is the first time that a Markovian environment is constructed in the end of Section 2 by means of useful information which arises from the rate volatility of the processes that the customers rent and return the bikes within one period (i.e., one day), where a fluctuating law of three peaks in the bike-rented (or bike-returned) processes is refined from the practical data of the tagged station of the bike sharing system, e.g. see Figure 1. On the other hand, the user's finite waiting rooms are added into some stations, and they enhance flexibility and ability of the bike sharing system such that the probability of problematic stations is reduced greatly. Thus the QoS of the bike sharing system can be promoted effectively by means of adding the user's finite waiting rooms, e.g., see Figures 9 and 10 for some numerical analysis. Note that the user's finite waiting rooms were first introduced and discussed by Leurent [20] through the $\mathrm{M} / \mathrm{M} / 1 / \mathrm{C}+\mathrm{K}$ queue in which only one isolated station is observed and analyzed, and the results obtained from the isolated station were used to provide a coarse-grained approximation for performance evaluation of the bike sharing system. Differently from Leurent [20], this paper analyzes a total network of the bike sharing system by means of the mean-field theory, where the nodes with finite waiting rooms may have a variety of weak interactions. The third contribution is to use some numerical examples to show effectiveness and computability of this mean-field matrixanalytic method, and to show how some key parameters influence performance measures of this bike sharing system. Therefore, we gain new insights on understanding nonlinear dynamics, inhomogeneous nature and interesting performance of the bike sharing systems, and hope the methodology and results given in this paper are applicable in the study of more general large-scale bike sharing systems.

The remainder of this paper is organized as follows. In Section 2, we describe a large-scale bike sharing system with $N$ identical stations and with user's finite waiting rooms under Markovian environment. Furthermore, we provide a method to construct a Markovian environment by means of a fluctuating rate law of three peaks within a period, which well expresses time-inhomogeneity and asymmetry of the processes that the customers rent and return the bikes. In Section 3, we first introduce an empirical measure process to express the states of this bike sharing system. Then we use a probability- 
analytic method to establish a necessary reference system: A time-inhomogeneous queue $\operatorname{MAP}(t) / \operatorname{MAP}(t) / 1 / \mathrm{K}+2 \mathrm{~L}+1$ by means of the mean-field theory. This help us to set up a block-structured system of mean-field equations. In Section 4, we apply the martingale limit theory to prove the asymptotic independence (or propagation of chaos) of the bike sharing system. In Section 5, we discuss the fixed point of the block-structured system of limiting mean-field equations, and provide a nonlinear QBD process to compute the fixed point. Furthermore, we study the limiting interchangeability as $N \rightarrow \infty$ and $t \rightarrow+\infty$. In Section 6, we give six numerical examples to investigate the performance measures, and show how some key parameters influence system performance. Some concluding remarks are given in Section 7.

\section{Model Description}

In this section, we describe a large-scale bike sharing system with $N$ identical stations and with user's finite waiting rooms under Markovian environment, and list operations mechanism, system parameters, model notation and necessary interpretation. Furthermore, we give a detailed discussion on how to construct a Markovian environment by means of useful information arose from the rate volatility of the process that the customers rent and return the bikes within one period (i.e., one day), where a fluctuating law of three peaks in the bike-rented (or bike-returned) processes is refined according to practical dynamics of the bike sharing systems.

In a bike sharing system, a customer first arrives at a station, rents a bike, and uses it for a while; then he returns the bike to a destination station. Once the customer finishes his trip and returns the bike to a station, he immediately leaves the bike sharing system. Based on this, Li et al. 26] first described a practical bike sharing system as a complicated closed queueing network with virtual customers (bikes) and two classes of virtual nodes (stations and roads). Since then, subsequent papers have been published on this theme. Li et al. [27] extended the model in [26] from two key factors: Markovian arrival processes and an irreducible road graph; while Li et al. 29] gave fluid and diffusion limits of the bike sharing system with renewal user arrivals and general riding-bike times.

Although it is seen from the closed queueing networks that the stationary probability of problematic stations can formally be computed by means of the product-form solution of the joint stationary distribution of queue lengths, there still exist some calculable dif- 
ficulties which arise from a rather complicated expression of the routing matrix of the corresponding virtual closed queueing network. Therefore, it is not only important for theoretic investigations but also necessary for engineering applications to provide some effective approximate techniques, for example, the mean-field theory of the bike sharing systems. Note that the mean-field theory has a key advantage that focuses on analyzing only one node with mean-field parameters whose basic information is sourced from the weak interactions among all the nodes of a network system. Thus performance measures of this node with mean-field parameters can be obtained easily, and they are used to well approximate that of the total network system. Thus the mean-field results can be used to show how some key system parameters influence the stationary probability of problematic stations so that the QoS of the bike sharing system can be evaluated from such a mean-field approximation.

Based on the above analysis, this paper extends the mean-field theory to be able to deal with more general bike sharing systems, and further provides a novel mean-field matrix-analytic method in the study of bike sharing systems. To this end, we describe a large-scale bike sharing system, and list its operations mechanism, system parameters, model notation and necessary interpretation as follows:

(1) The $N$ identical stations: To use the mean-field theory, we assume that the large-scale bike sharing system contains $N$ identical stations; and at the initial time $t=0$, every station has $C$ bikes and $K$ parking places, in which $1 \leq C \leq K<\infty$. Every station continuously operates through either renting a bike or returning a bike, so the number of bikes in a tagged station can be regarded as a queueing process.

(2) Adding user's finite waiting rooms to the stations: To decrease the probability of problematic stations when customers are sufficient in this system, it is an efficient technique to add a user's finite waiting room at each station. The waiting room have $L$ waiting places, each of which is occupied by only a customer when either he can not rent a bike from the tagged station or he can not return his bike to the tagged station. In general, each finite waiting room has two useful purposes: (a) When a customer arrives at an empty station in which no bike can be rented, either he enters a waiting place in order to wait for a future available bike with probability $\alpha \in[0,1]$, or he immediately leaves the bike sharing system with probability $1-\alpha$. (b) When a riding-bike customer completes his trip and enters a full station in which no empty parking place is available, either he enters a waiting place in order to wait for a future available parking place with probability 
$\beta \in[0,1]$, or he immediately rides his bike to another station in order to return the bike with probability $1-\beta$. Here, we must explain that the riding-bike customer must return his bike to any station, and then he can leave the bike sharing system, because each bike is an indispensable public equipment and cannot be lost or become a personal property.

(3) The Markovian environment: In this bike sharing system, the arrival and travel processes are influenced (or controlled) by a Markovian environment, which is a continuous-time irreducible positive-recurrent Markov process whose infinitesimal generator of size $m$ is given by

$$
\mathbf{W}=\left(\begin{array}{cccc}
w_{1,1} & w_{1,2} & \cdots & w_{1, m} \\
w_{2,1} & w_{2,2} & \cdots & w_{2, m} \\
\vdots & \vdots & \ddots & \vdots \\
w_{m, 1} & w_{m, 2} & \cdots & w_{m, m}
\end{array}\right)
$$

where $w_{i, i}<0$ for $1 \leq i \leq m ; w_{i, j} \geq 0$ for $1 \leq i, j \leq m$ and $i \neq j ; \sum_{j=1}^{m} w_{i, j}=0$ for $1 \leq i \leq m$. At the same time, we denote by $\theta$ the stationary probability vector of the Markov process $\mathbf{W}$, that is, $\theta \mathbf{W}=0$ and $\theta e=1$, where $e$ is a column vector of ones. Based on the Markov process $\mathbf{W}$, now we describe the arrival processes and the riding-bike times as follows:

(3.1) The arrival processes: If the Markovian environment is at State $j$, then the arrivals of customers at the bike sharing system from outside are a Poisson process with arrival rate $N \lambda_{j}$ for $1 \leq j \leq m$.

(3.2) The riding-bike times: If the Markovian environment is at State $j$, then the riding-bike time that a customer rides a bike from one station to another is exponential with travel rate $\mu_{j}$ for $1 \leq j \leq m$.

(4) The leaving principle: Once a customer finishes his trip and returns his bike to any station, he immediately leaves the bike sharing system.

We assume that all the random variables defined above are independent of each other.

When observing a tagged station in the bike sharing system, the finite waiting room and the Markovian environment play a key role in queueing analysis of this tagged station. To explain this, the queueing structure of the tagged station is depicted in Figure 1.

Remark 1 On the one hand, the assumption of the $N$ identical stations is used to guarantee applicability of the mean-field theory (that is, the multi-dimensional Markov process 


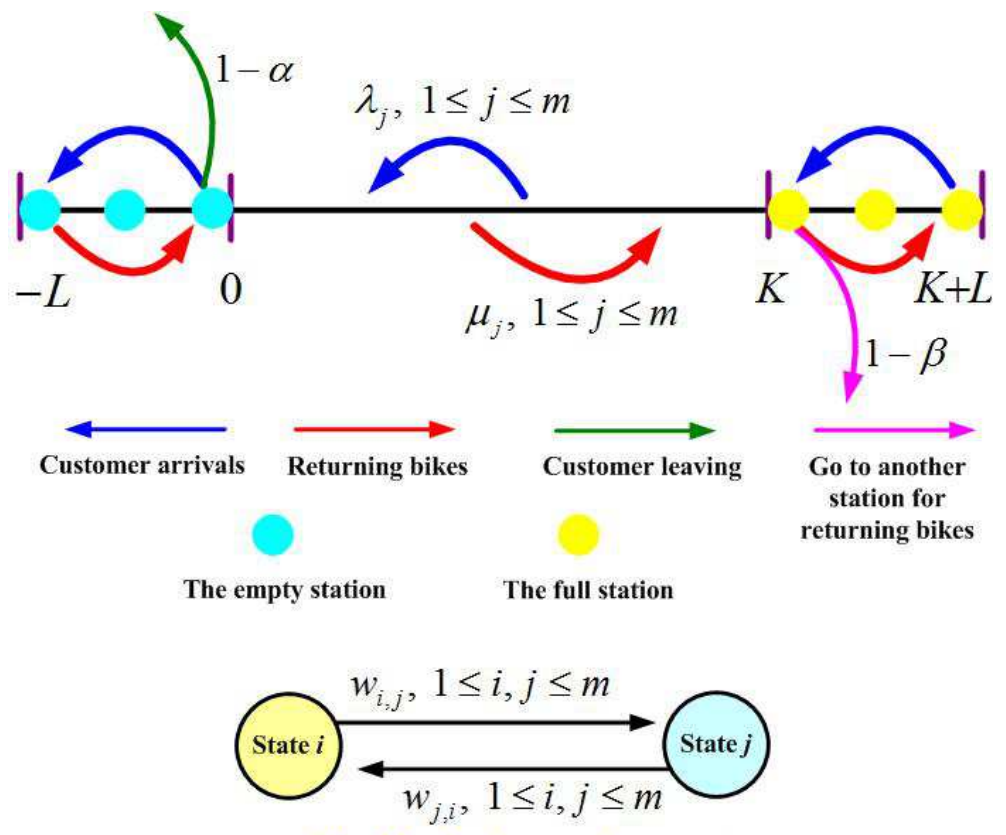

The Markovian environment

Figure 1: The queueing structure of any station in this bike-sharing system

is exchangeable). On the other hand, from a practical point of view, the stations in a major city are also designed as almost the same, for example, Hangzhou has over 4000 stations, and each station contains about 30 bikes.

Remark 2 In some major cities, there are always many bikes and customers distributed at various stations to support the short trips. To improve the quality of service (or to decrease the probability of problematic stations), an adscititious waiting room designed to add at each station of a bike sharing system is always effective and useful. Leurent [20] first proposed such an idea of adscititious waiting rooms, and discussed the queueing process of only one isolated station by means of the $M / M / 1 / C$ queue. Differently from Leurent [20], this paper applies the mean-field theory to analyse such a network system of the bike sharing system with user's waiting rooms, and then numerically compares performance measures of the bike sharing systems with or without user's finite waiting rooms, e.g., see Figure 9 for more details.

In the remainder of this section, we give an interesting idea that constructs a Markovian environment with seven states to be able to well express the time-inhomogeneity and 


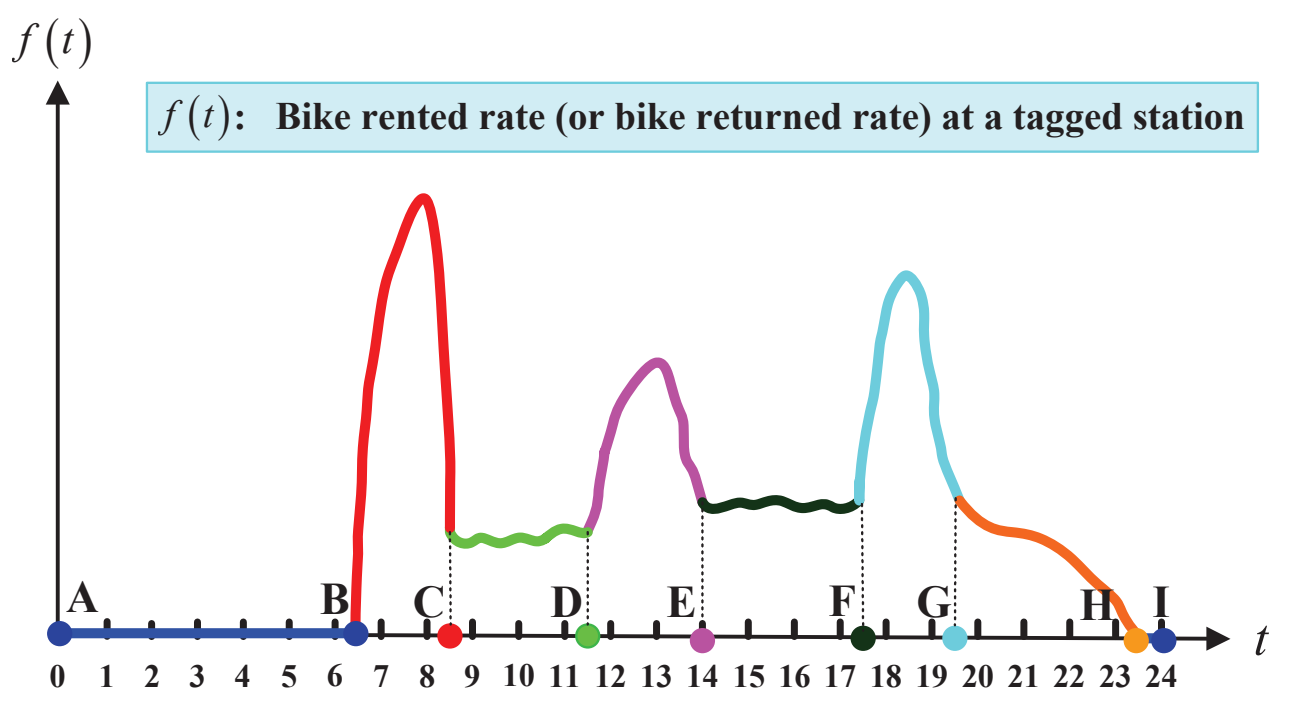

A law of three peaks of the rate $f(t)$ during one period (i.e., a day)

Figure 2: A fluctuating law of three peaks for bike rented (or returned) rates within a day asymmetry of the processes that the customers rent and return the bikes. To our best knowledge, it is the first time that a Markovian environment is constructed by means of useful information which arises from the rate volatility of the processes that the customers rent and return the bikes within a day, where a fluctuating law of three peaks in bike rented (or returned) rates within a day is depicted in Figure 2.

Based on Figure 2, we segment 24 hours of one day into 7 parts as follows:

$$
\begin{array}{rlrl}
\text { Part one } & =[0,6.5) \cup[23.5,24), & \text { Part two }=[6.5,8.5), \\
\text { Part three } & =[8.5,11.5), & & \text { Part four }=[11.5,14), \\
\text { Part five } & =[14,17.5), & \text { Part six }=[17.5,19.5), \\
\text { Part seven } & =[19.5,23.5) . & &
\end{array}
$$

Since the seven segmented parts within 24 hours demonstrate a stable regular structure of periodical dynamics of the bike sharing systems, Part $i$ may be regarded as State $i$ of a Markovian environment. Note that the Markovian environment can be expressed by an irreducible Markov process whose state transition relations are described as

State $1 \rightarrow$ State $2 \rightarrow$ State $3 \rightarrow$ State $4 \rightarrow$ State $5 \rightarrow$ State $6 \rightarrow$ State $7 \rightarrow$ State 1 . 
Thus the infinitesimal generator of the Markovian environment is given by

$$
\mathbf{W}=\left(\begin{array}{ccccccc}
-x_{1} & x_{1} & & & & & \\
& -x_{2} & x_{2} & & & & \\
& & -x_{3} & x_{3} & & & \\
& & & -x_{4} & x_{4} & & \\
& & & & -x_{5} & x_{5} & \\
& & & & & -x_{6} & x_{6} \\
x_{7} & & & & & & -x_{7}
\end{array}\right) .
$$

To compute the undetermined numbers $x_{i}$ for $1 \leq i \leq 7$, we first take the stationary probability vector $\theta=\left(\theta_{1}, \theta_{2}, \ldots, \theta_{7}\right)$ according to the time length ratios of the seven segmented parts within 24 hours. Thus it is seen from Figure 2 that

$$
\theta_{1}=\frac{7}{24}, \theta_{2}=\frac{2}{24}, \theta_{3}=\frac{3}{24}, \theta_{4}=\frac{2.5}{24}, \theta_{5}=\frac{3.5}{24}, \theta_{6}=\frac{2}{24}, \theta_{7}=\frac{4}{24} .
$$

Let $x_{7}=1$. Then it follows from $\theta \mathbf{W}=0$ that

$$
x_{i}=\frac{\theta_{7}}{\theta_{i}}, \quad 1 \leq i \leq 7
$$

Now, we provide an average approximate method to determine the bike rented and returned rates which are controlled by the states of the Markovian environment. To this end, let $f_{\text {rent }}(t)$ and $f_{\text {return }}(t)$ be the instantaneous rates of the bike rented and returned processes at time $t \geq 0$, respectively. Note that each of the two functions $f_{\text {rent }}(t)$ and $f_{\text {return }}(t)$ is referred to the fluctuating law of three peaks depicted in Figure 2, or they can be approximately evaluated by means of the associated data collected from system 
operations. Based on this, we set up

$$
\begin{aligned}
& \lambda_{1}=0, \mu_{1}=0 ; \\
& \lambda_{2}=\frac{1}{2} \int_{6.5}^{8.5} f_{\text {rent }}(t) \mathrm{d} t, \mu_{2}=\frac{1}{2} \int_{6.5}^{8.5} f_{\text {return }}(t) \mathrm{d} t ; \\
& \lambda_{3}=\frac{1}{3} \int_{8.5}^{11.5} f_{\text {rent }}(t) \mathrm{d} t, \mu_{3}=\frac{1}{3} \int_{8.5}^{11.5} f_{\text {return }}(t) \mathrm{d} t ; \\
& \lambda_{4}=\frac{1}{2.5} \int_{11.5}^{14} f_{\text {rent }}(t) \mathrm{d} t, \mu_{4}=\frac{1}{2.5} \int_{11.5}^{14} f_{\text {return }}(t) \mathrm{d} t ; \\
& \lambda_{5}=\frac{1}{3.5} \int_{14}^{17.5} f_{\text {rent }}(t) \mathrm{d} t, \mu_{5}=\frac{1}{3.5} \int_{14}^{17.5} f_{\text {return }}(t) \mathrm{d} t ; \\
& \lambda_{6}=\frac{1}{2} \int_{17.5}^{19.5} f_{\text {rent }}(t) \mathrm{d} t, \mu_{6}=\frac{1}{2} \int_{17.5}^{19.5} f_{\text {return }}(t) \mathrm{d} t ; \\
& \lambda_{7}=\frac{1}{4} \int_{19.5}^{23.5} f_{\text {rent }}(t) \mathrm{d} t, \mu_{7}=\frac{1}{4} \int_{19.5}^{23.5} f_{\text {return }}(t) \mathrm{d} t .
\end{aligned}
$$

In general, the two functions $f_{\text {rent }}(t)$ and $f_{\text {return }}(t)$ can approximately be given by means of some statistical models to deal with the practical data in a tagged station of the bike sharing system.

Remark 3 By using the fluctuating law of three peaks depicted in Figure 2, we choose seven different states to construct a Markovian environment, which is related to real-time dynamics of the bike sharing system. Similarly, we may also set up a fluctuating law of $n$ peaks to construct a Markovian environment of $2 n+1$ states. Note that the approximate accuracy of such modeling can be improved effectively as the number $n$ increases. On the other hand, it is necessary to mention that the Markovian environment constructed from a fluctuating law of $n$ peaks will be very useful in the study of stochastic periodic systems because the difficult periodic dynamic system is transformed to an easy Markov system, for example, ride sharing systems, healthcare systems, transportation networks, periodic retail systems, wind power system and so forth.

\section{Mean-Field Equations}

In this section, we first introduce an empirical measure process to express the states of this bike sharing system. Then we provide a probability-analytic method, combining with the mean-field theory, to establish a necessary reference system: A time-inhomogeneous queue $\operatorname{MAP}(t) / \operatorname{MAP}(t) / 1 / \mathrm{K}+2 \mathrm{~L}+1$. This help us to set up a block-structured system of mean-field equations. 
Now, we introduce an empirical measure process to express the states of this bike sharing system.

Let $X_{i}^{(N)}(t)$ and $J(t)$ be the number of customers in Station $i$ and the state of the Markovian environment at time $t$, respectively. Then $\mathbf{X}=\left\{\left(X_{1}^{(N)}(t), X_{2}^{(N)}(t), \ldots, X_{N}^{(N)}(t)\right.\right.$; $J(t)): t \geq 0\}$ is an $N m$-dimensional Markov process due to the assumptions on the Poisson arrivals, the exponential travel times and the Markovian environment. Note that analysis of such an $\mathrm{Nm}$-dimensional Markov process is always difficult due to the "State Space Explosion". Therefore, it is necessary to introduce an empirical measure process.

For the Markov process $\mathbf{X}=\left\{\left(X_{1}^{(N)}(t), X_{2}^{(N)}(t), \ldots, X_{N}^{(N)}(t) ; J(t)\right): t \geq 0\right\}$, the empirical measure is defined as

$$
Y_{k, j}^{(N)}(t)=\frac{1}{N} \sum_{n=1}^{N} \mathbf{1}_{\left\{X_{n}^{(N)}(t)=k, J(t)=j\right\}},
$$

where $\mathbf{1}_{\{\bullet\}}$ is an indicative function. Obviously, $Y_{k, j}^{(N)}(t)$ denotes the fraction of stations with $k$ bikes and with the Markovian environment be at State $j$ at time $t$. It is easy to see that for $-L \leq k \leq K+L$,

$$
0 \leq Y_{k, j}^{(N)}(t) \leq \sum_{j=1}^{m} Y_{k, j}^{(N)}(t) \leq \sum_{k=-L}^{K+L} \sum_{j=1}^{m} Y_{k, j}^{(N)}(t)=1 .
$$

Let

$$
\mathbf{Y}_{k}^{(N)}(t)=\left(Y_{k, 1}^{(N)}(t), Y_{k, 2}^{(N)}(t), \ldots, Y_{k, m}^{(N)}(t)\right)
$$

and

$$
\mathbf{Y}^{(N)}(t)=\left(\mathbf{Y}_{-L}^{(N)}(t), \mathbf{Y}_{-L+1}^{(N)}(t), \ldots, \mathbf{Y}_{K+L-1}^{(N)}(t), \mathbf{Y}_{K+L}^{(N)}(t)\right)
$$

which is a row vector of size $(K+2 L+1) m$. Then $\left\{\mathbf{Y}^{(N)}(t): t \geq 0\right\}$ is an empirical measure Markov process whose state space is given by $\Omega=[0,1]^{(K+2 L+1) m}$.

To consider the empirical measure Markov process $\left\{\mathbf{Y}^{(N)}(t): t \geq 0\right\}$, we write

$$
y_{k, j}^{(N)}(t)=E\left[Y_{k, j}^{(N)}(t)\right], \quad-L \leq k \leq K+L, 1 \leq j \leq m,
$$

and

$$
\begin{gathered}
\mathbf{y}_{k}^{(N)}(t)=\left(y_{k, 1}^{(N)}(t), y_{k, 2}^{(N)}(t), \ldots, y_{k, m}^{(N)}(t)\right) \\
\mathbf{y}^{(N)}(t)=\left(\mathbf{y}_{-L}^{(N)}(t), \mathbf{y}_{-L+1}^{(N)}(t), \ldots, \mathbf{y}_{K+L-1}^{(N)}(t), \mathbf{y}_{K+L}^{(N)}(t)\right) .
\end{gathered}
$$

In what follows we will apply the mean-field theory to set up a block-structured system of mean-field equations whose purpose is to be able to numerically compute the key vector $\mathbf{y}^{(N)}(t)$. 


\subsection{A time-inhomogeneous $\operatorname{MAP}(t) / \mathrm{MAP}(t) / 1 / \mathrm{K}+2 \mathrm{~L}+1$ queue}

In the bike sharing system with $N$ identical stations and with Markovian environment, we define $\mathbf{Q}^{(N)}(t)$ as the number of bikes in a tagged station at time $t$. It is easy to see that if an outside customer arrives at the tagged station and rents a bike, then $\mathbf{Q}^{(N)}(t)$ decreases by one; while if a customer finishes his trip and returns a bike at the tagged station, $\mathbf{Q}^{(N)}(t)$ increases by one. Based on this, we can understand that the Markov process $\left\{\left(\mathbf{Q}^{(N)}(t), J(t)\right): t \geq 0\right\}$ is a QBD process, which is further shown to well correspond to a time-inhomogeneous $\operatorname{MAP}(t) / \mathrm{MAP}(t) / 1 / \mathrm{K}+2 \mathrm{~L}+1$ queue, where $\mathrm{MAP}(t)$ is an instantaneous Markov arrival process with a matrix descriptor $(C(t), D(t))$ of size $m$, e.g., see Subsections 8.2.5 and 8.2.6 of Chapter 8 in Li [21] for more details.

In the $\operatorname{MAP}(t) / \operatorname{MAP}(t) / 1 / \mathrm{K}+2 \mathrm{~L}+1$ queue, it is easy to understand that the customers are virtual from the bikes, that is, the bikes are the virtual customers. Thus a virtual customer's arrival is a bike returned to the tagged station; while a virtual customer's service completion is a bike rented from the tagged station. Thus, here we call arrival (or service) to be virtual arrival (or virtual service).

The following theorem provides expressions for the instantaneous virtual arrival rate $\xi_{l, j}^{(N)}(t)$ and the instantaneous virtual service rate $\eta_{k, j}^{(N)}(t)$ in this time-inhomogeneous queueing system. Note that the two instantaneous rates play a key role in our later study.

Theorem 1 In the time-inhomogeneous $M A P(t) / M A P(t) / 1 / K+2 L+1$ queue, we have

(a) the instantaneous virtual service rate is given by

$$
\eta_{k, j}^{(N)}(t)= \begin{cases}\lambda_{j}, & 1 \leq k \leq K+L, \quad 1 \leq j \leq m, \\ \lambda_{j} \alpha, & -(L-1) \leq k \leq 0, \quad 1 \leq j \leq m,\end{cases}
$$

which is independent of the number $N$. 
(b) For $1 \leq j \leq m$, the instantaneous virtual arrival rate is given by

$$
\xi_{l, j}^{(N)}(t)=\left\{\begin{array}{l}
\frac{\mu_{j}}{N}\left\{C+(N-1)\left[C-\sum_{k=1}^{K+L} k y_{k, j}^{(N)}(t)\right.\right. \\
\left.\left.+\sum_{k=K}^{K+L-1} \frac{(1-\beta) y_{k, j}^{(N)}(t)}{\left[1-(1-\beta) y_{k, j}^{(N)}(t)\right]^{2}}+\frac{y_{K+L, j}^{(N)}(t)}{\left[1-y_{K+L, j}^{(N)}(t)\right]^{2}}\right]\right\}, \quad-L \leq l \leq 0, \\
\frac{\mu_{j}}{N}\left\{C-l+(N-1)\left[C-\sum_{k=1}^{K+L} k y_{k, j}^{(N)}(t)\right.\right. \\
\left.\left.+\sum_{k=K}^{K+L-1} \frac{(1-\beta) y_{k, j}^{(N)}(t)}{\left[1-(1-\beta) y_{k, j}^{(N)}(t)\right]^{2}}+\frac{y_{K+L, j}^{(N)}(t)}{\left[1-y_{K+L, j}^{(N)}(t)\right]^{2}}\right]\right\}, \quad 1 \leq l \leq C-1, \\
\frac{\mu_{j}}{N}\left\{( N - 1 ) \left[C-\sum_{k=1}^{K+L} k y_{k, j}^{(N)}(t)\right.\right. \\
\left.\left.+\sum_{k=K}^{K+L-1} \frac{(1-\beta) y_{k, j}^{(N)}(t)}{\left[1-(1-\beta) y_{k, j}^{(N)}(t)\right]^{2}}+\frac{y_{K+L, j}^{(N)}(t)}{\left[1-y_{K+L, j}^{(N)}(t)\right]^{2}}\right]\right\}, \quad C \leq l \leq K-1, \\
\beta \frac{\mu_{j}}{N}\left\{( N - 1 ) \left[C-\sum_{k=1}^{K+L} k y_{k, j}^{(N)}(t)\right.\right. \\
\left.\left.+\sum_{k=K}^{K+L-1} \frac{(1-\beta) y_{k, j}^{(N)}(t)}{\left[1-(1-\beta) y_{k, j}^{(N)}(t)\right]^{2}}+\frac{y_{K+L, j}^{(N)}(t)}{\left[1-y_{K+L, j}^{(N)}(t)\right]^{2}}\right]\right\}, \quad K \leq l \leq K+L-1 .
\end{array}\right.
$$

Proof: The proof of (2). When a customer arrives at a tagged station, there exist two different cases:

Case (a) If the station has at least one bike (that is, $1 \leq k \leq K+L$ ), then he immediately rents a bike and leaves the station, that is, the virtual service is completed. Hence $\eta_{k, j}^{(N)}(t)=\lambda_{j}$ for $1 \leq j \leq m$.

Case (b) If the station has no bike (that is, $-L+1 \leq k \leq 0$ ), then he has two choices: he directly leaves this system with the probability $1-\alpha$; or he enters a waiting place with the probability $\alpha$ in order to wait for renting a future available bike. Clearly, the virtual service has not been completed yet but this also leads to the shortage of virtual customers (or bikes). In this case, the rate $\lambda_{j} \alpha$ expresses the transition speed of that the number of bikes at the tagged station from $k$ to $k-1$ for $-(L-1) \leq k \leq 0$. Thus $\eta_{k, j}^{(N)}(t)=\lambda_{j} \alpha$ for $1 \leq j \leq m$, and it is independent of the number $k=-(L-1),-(L-2), \ldots, 1,0$.

Based on Cases (a) and (b), when the Markovian environment $J(t)=j$, we have

$$
\eta_{k, j}^{(N)}(t)= \begin{cases}\lambda_{j}, & 1 \leq k \leq K+L, \quad 1 \leq j \leq m, \\ \lambda_{j} \alpha, & -(L-1) \leq k \leq 0, \quad 1 \leq j \leq m,\end{cases}
$$

which is independent of $k=-(L-1),-(L-2), \ldots, K+L-1, K+L$.

The proof of (3). The proof of (3) is a bit complicated due to applications of the mean-field theory. Note that Figure 3 describes the state transitions of the process that 


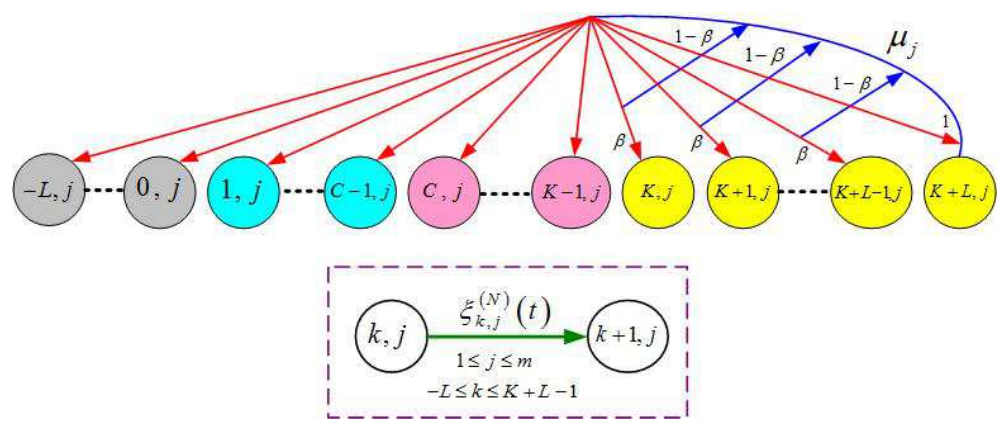

Figure 3: The state transition relation of the queueing process in the tagged station

the bikes are returned at the tagged station. Since the bikes can not be returned to a full station, either the user enters a waiting place in order to wait for a future available parking place with probability $\beta$, or he immediately rides his bike to another station to find an available parking place with probability $1-\beta$. Based on this, we give the instantaneous virtual arrival rate $\xi_{l, j}^{(N)}(t)$ by means of a probability-analytic method as follows:

$$
\xi_{l, j}^{(N)}(t)=\frac{1}{N} \cdot \mu_{j} \cdot \text { the number of bikes ridden on all the roads at time } t \text {. }
$$

Note that the number of bikes ridden on all the roads contains two parts: (i) The number $n_{1}$ of bikes ridden from the tagged station is given by

$$
n_{1}= \begin{cases}C, & -L \leq l \leq 0 \\ C-l, & 1 \leq l \leq C-1 \\ 0, & C \leq l \leq K+L-1\end{cases}
$$

and (ii) the number of bikes having been ridden from the other $N-1$ station is given by

$(N-1)$ [the average number of bikes directly ridden from one of the $N-1$ stations

+ the average number of bikes which can not be returned to a full station with at least

two retries].

By using the mean-field theory, the average number of bikes ridden from the tagged station is given by $C-\sum_{k=1}^{K+L} k y_{k, j}^{(N)}(t)$. While the average number of bikes which can not be returned to a full station with at least two retries is given a detailed computation below.

Based on the above analysis, our computation for deriving the instantaneous virtual arrival rate $\xi_{l, j}^{(N)}(t)$ is divided into the following four cases. 
Case (a): When $-L \leq l \leq 0,1 \leq j \leq m$, we need to study three different classes for the initial distribution of bikes in the tagged station. Note that in the last two classes, users who arrive at a full station can not return their bikes at the station.

Class-1: The initial $C$ bikes in the tagged station are all rented on the roads. Using the mean-field theory, we get that the average number of bikes rented on the road from the other $N-1$ stations is given by

$$
(N-1)\left[C-\sum_{k=1}^{K+L} k y_{k, j}^{(N)}(t)\right]
$$

where $\sum_{k=1}^{K+L} k y_{k, j}^{(N)}(t)$ is the average number of bikes parked in the tagged station. Thus, the average number of bikes rented on the roads from the $N$ stations is given by

$$
C+(N-1)\left[C-\sum_{k=1}^{K+L} k y_{k, j}^{(N)}(t)\right] .
$$

Class-2: A customer finishes his trip and arrives at a tagged station in which there are $k$ bikes for $K \leq k \leq K+L-1$. It is clear that the tagged station is full so that the customer has to re-ride the bike in order to return the bike to another station with the probability $1-\beta$. The average number of such re-riding bike is given by

$$
\begin{aligned}
& \sum_{k=K}^{K+L-1}\left\{(1-\beta) y_{k, j}^{(N)}(t)+2\left[(1-\beta) y_{k, j}^{(N)}(t)\right]^{2}+3\left[(1-\beta) y_{k, j}^{(N)}(t)\right]^{3}+\cdots\right\} \\
= & \sum_{k=K}^{K+L-1} \frac{(1-\beta) y_{k, j}^{(N)}(t)}{\left[1-(1-\beta) y_{k, j}^{(N)}(t)\right]^{2}},
\end{aligned}
$$

where $n\left[(1-\beta) y_{k, j}^{(N)}(t)\right]^{n}$ is the average number of re-riding bikes of $n$ customers, and $x+2 x^{2}+3 x^{3}+\cdots=x /(1-x)^{2}$.

Class-3: A customer finishes his trip and arrives at a tagged station in which there are $K+L$ bikes. In this case, there is neither a bike-parking place nor a user-waiting place, hence the customer has to re-ride the bike in order to return the bike at another station with the probability 1 . The average number of such re-riding bikes is given by

$$
y_{K+L, j}^{(N)}(t)+2\left[y_{K+L, j}^{(N)}(t)\right]^{2}+3\left[y_{K+L, j}^{(N)}(t)\right]^{3}+\cdots=\frac{y_{K+L, j}^{(N)}(t)}{\left[1-y_{K+L, j}^{(N)}(t)\right]^{2}} .
$$

Summarizing the above analysis, the instantaneous virtual arrival rate is given by

$\xi_{l, j}^{(N)}(t)=\frac{\mu_{j}}{N}\left\{C+(N-1)\left[C-\sum_{k=1}^{K+L} k y_{k, j}^{(N)}(t)+\sum_{k=K}^{K+L-1} \frac{(1-\beta) y_{k, j}^{(N)}(t)}{\left[1-(1-\beta) y_{k, j}^{(N)}(t)\right]^{2}}+\frac{y_{K+L, j}^{(N)}(t)}{\left[1-y_{K+L, j}^{(N)}(t)\right]^{2}}\right]\right\}$. 
Case (b): When $1 \leq l \leq C-1$, the only difference of our derivation from Case (a) is to replace the initial $C$ bikes by the initial $C-l$ bikes in the tagged station. Thus we get $\xi_{l, j}^{(N)}(t)=\frac{\mu_{j}}{N}\left\{C-l+(N-1)\left[C-\sum_{k=1}^{K+L} k y_{k, j}^{(N)}(t)+\sum_{k=K}^{K+L-1} \frac{(1-\beta) y_{k, j}^{(N)}(t)}{\left[1-(1-\beta) y_{k, j}^{(N)}(t)\right]^{2}}+\frac{y_{K+L, j}^{(N)}(t)}{\left[1-y_{K+L, j}^{(N)}(t)\right]^{2}}\right]\right\}$.

Case (c): When $C \leq l \leq K-1$, the only difference of our derivation from Case (a) is that the initial $C$ bikes in this station are all parked in the tagged station. Hence we obtain

$\xi_{l, j}^{(N)}(t)=\frac{\mu_{j}}{N}\left\{(N-1)\left[C-\sum_{k=1}^{K+L} k y_{k, j}^{(N)}(t)+\sum_{k=K}^{K+L-1} \frac{(1-\beta) y_{k, j}^{(N)}(t)}{\left[1-(1-\beta) y_{k, j}^{(N)}(t)\right]^{2}}+\frac{y_{K+L, j}^{(N)}(t)}{\left[1-y_{K+L, j}^{(N)}(t)\right]^{2}}\right]\right\}$.

Case (d): When $K \leq l \leq K+L-1$, the only difference of our derivation from Case (c) is that when a customer finishes his trip and arrives at the tagged station, he enters the waiting places in order to wait for an empty parking place with the probability $\beta$. This gives

$\xi_{l, j}^{(N)}(t)=\beta \frac{\mu_{j}}{N}\left\{(N-1)\left[C-\sum_{k=1}^{K+L} k y_{k, j}^{(N)}(t)+\sum_{k=K}^{K+L-1} \frac{(1-\beta) y_{k, j}^{(N)}(t)}{\left[1-(1-\beta) y_{k, j}^{(N)}(t)\right]^{2}}+\frac{y_{K+L, j}^{(N)}(t)}{\left[1-y_{K+L, j}^{(N)}(t)\right]^{2}}\right]\right\}$.

Summarizing the above four cases, we obtain all the expressions given in (3). This completes the proof.

\subsection{A block-structured system of mean-field equations}

Based on the time-inhomogeneous $\operatorname{MAP}(t) / \mathrm{MAP}(t) / 1 / \mathrm{K}+2 \mathrm{~L}+1$ queue, it is convenient to describe the time-inhomogeneous QBD process $\left\{\left(\mathbf{Q}^{(N)}(t), J(t)\right): t \geq 0\right\}$ whose state transition relation is depicted in Figure 4. At the same time, a useful relation related to (1) for understanding the state probability distribution of the QBD process is given by

$$
y_{k, j}^{(N)}(t)=P\left\{\mathbf{Q}^{(N)}(t)=k, J(t)=j\right\}, \quad-L \leq k \leq K+L, 1 \leq j \leq m .
$$




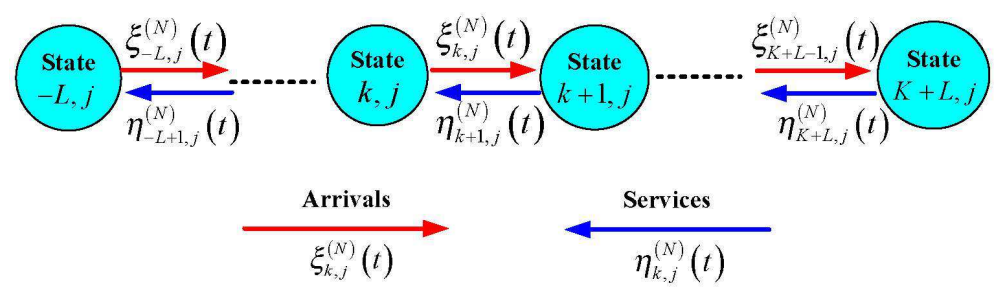

Figure 4: The state transitions of the time-inhomogeneous QBD process

Based on the instantaneous virtual arrival rate $\xi_{l, j}^{(N)}(t)$, we set that for $-L \leq k \leq K+L-1$

$$
\begin{gathered}
\Psi_{k}^{(N)}(t)=\left(\begin{array}{cccc}
0 & \xi_{k, 1}^{(N)}(t) w_{1,2} & \cdots & \xi_{k, 1}^{(N)}(t) w_{1, m} \\
\xi_{k, 2}^{(N)}(t) w_{2,1} & 0 & \cdots & \xi_{k, 2}^{(N)}(t) w_{2, m} \\
\vdots & \vdots & \ddots & \vdots \\
\xi_{k, m}^{(N)}(t) w_{m, 1} & \xi_{k, m}^{(N)}(t) w_{m, 2} & \cdots & 0
\end{array}\right), \\
\widehat{\Psi}_{k}^{(N)}(t)=\operatorname{diag}\left(\xi_{k, 1}^{(N)}(t) w_{1,1}, \xi_{k, 2}^{(N)}(t) w_{2,2}, \ldots, \xi_{k, m}^{(N)}(t) w_{m, m}\right)
\end{gathered}
$$

Similarly, it is easy to see from the instantaneous virtual service rate $\eta_{k, j}^{(N)}(t)$ that for $-L+1 \leq k \leq 0$

$$
\begin{gathered}
\Phi_{k}^{(N)}(t)=\left(\begin{array}{cccc}
0 & \lambda_{1} \alpha w_{1,2} & \cdots & \lambda_{1} \alpha w_{1, m} \\
\lambda_{2} \alpha w_{2,1} & 0 & \cdots & \lambda_{2} \alpha w_{2, m} \\
\vdots & \vdots & \ddots & \vdots \\
\lambda_{m} \alpha w_{m, 1} & \lambda_{m} \alpha w_{m, 2} & \cdots & 0
\end{array}\right) \stackrel{\text { Def }}{=} \Phi(\alpha), \\
\widehat{\Phi}_{k}^{(N)}(t)=\operatorname{diag}\left(\lambda_{1} \alpha w_{1,1}, \lambda_{2} \alpha w_{2,2}, \ldots, \lambda_{m} \alpha w_{m, m}\right) \stackrel{\text { Def }}{=} \widehat{\Phi}(\alpha) ;
\end{gathered}
$$

while for $1 \leq k \leq K+L$

$$
\Phi_{k}^{(N)}(t)=\Phi(1)
$$

and

$$
\widehat{\Phi}_{k}^{(N)}(t)=\widehat{\Phi}(1),
$$

both of which are due to $\alpha=1$ for $1 \leq k \leq K+L$.

For the time-inhomogeneous QBD process $\left\{\left(\mathbf{Q}^{(N)}(t), J(t)\right): t \geq 0\right\}$, it follows from Figure 4 that the vector $\mathbf{y}^{(N)}(t)=\left(\mathbf{y}_{-L}^{(N)}(t), \mathbf{y}_{-L+1}^{(N)}(t), \ldots, \mathbf{y}_{K+L-1}^{(N)}(t), \mathbf{y}_{K+L}^{(N)}(t)\right)$ satisfies a block-structured system of mean-field (or ordinary differential) equations as follows:

$$
\frac{\mathrm{d}}{\mathrm{d} t} \mathbf{y}_{-L}^{(N)}(t)=\mathbf{y}_{-L}^{(N)}(t) \widehat{\Psi}_{-L}^{(N)}(t)+\mathbf{y}_{-L+1}^{(N)}(t) \Phi_{-L+1}^{(N)}(t)
$$


for $-L+1 \leq k \leq K+L-1$

$$
\begin{aligned}
\frac{\mathrm{d}}{\mathrm{d} t} \mathbf{y}_{k}^{(N)}(t)= & \mathbf{y}_{k-1}^{(N)}(t) \Psi_{k-1}^{(N)}(t)+\mathbf{y}_{k}^{(N)}(t)\left[\widehat{\Psi}_{k}^{(N)}(t)+\widehat{\Phi}_{k}^{(N)}(t)\right]+\mathbf{y}_{k+1}^{(N)}(t) \Phi_{k+1}^{(N)}(t), \\
& \frac{\mathrm{d}}{\mathrm{d} t} \mathbf{y}_{K+L}^{(N)}(t)=\mathbf{y}_{K+L}^{(N)}(t) \widehat{\Phi}_{K+L}^{(N)}(t)+\mathbf{y}_{K+L-1}^{(N)}(t) \Psi_{K+L-1}^{(N)}(t),
\end{aligned}
$$

with the boundary condition

$$
\sum_{k=-L}^{K+L} \mathbf{y}_{k}^{(N)}(t) e=1
$$

and with the initial condition

$$
\mathbf{y}_{k}^{(N)}(0)=g_{k}, \quad-L \leq k \leq K+L,
$$

and

$$
\begin{gathered}
g_{k}=\left(g_{k, 1}, g_{k, 2}, \ldots, g_{k, m}\right), \\
\mathbf{g}=\left(g_{-L}, g_{-(L-1)}, \cdots, g_{K+L-1}, g_{K+L}\right)
\end{gathered}
$$

is a probability vector of size $(K+2 L+1) m$.

For convenience of description, we write the mean-field equations (44) to (8) into a matrix version as follows:

$$
\frac{\mathrm{d}}{\mathrm{d} t} \mathbf{y}^{(N)}(t)=\mathbf{y}^{(N)}(t) \mathbf{V}_{\mathbf{y}^{(N)}(t)},
$$

with the boundary and initial conditions

$$
\mathbf{y}^{(N)}(t) e=1, \quad \mathbf{y}^{(N)}(0)=\mathbf{g}
$$

where

$$
\begin{aligned}
& \mathbf{V}_{\mathbf{y}^{(N)}(t)}=\left(\begin{array}{cccc}
A_{1,1} & A_{1,2} & & \\
A_{2,1} & A_{2,2} & A_{2,3} & \\
& A_{3,2} & A_{3,3} & A_{3,4} \\
& & A_{4,3} & A_{4,4}
\end{array}\right), \\
& \Delta_{k}^{(N)}(t)=\widehat{\Phi}_{k}^{(N)}(t)+\widehat{\Psi}_{k}^{(N)}(t), \quad-(L-1) \leq k \leq K+L-1, \\
& A_{1,1}=\left(\begin{array}{ccccc}
\widehat{\Psi}_{-L}^{(N)}(t) & \Psi_{-L}^{(N)}(t) & & & \\
\Phi_{-(L-1)}^{(N)}(t) & \Delta_{-(L-1)}^{(N)}(t) & \Psi_{-(L-1)}^{(N)}(t) & & \\
& \ddots & \ddots & \ddots & \\
& & \Phi_{-1}^{(N)}(t) & \Delta_{-1}^{(N)}(t) & \Psi_{-1}^{(N)}(t) \\
& & & \Phi_{0}^{(N)}(t) & \Delta_{0}^{(N)}(t)
\end{array}\right) \text {, }
\end{aligned}
$$




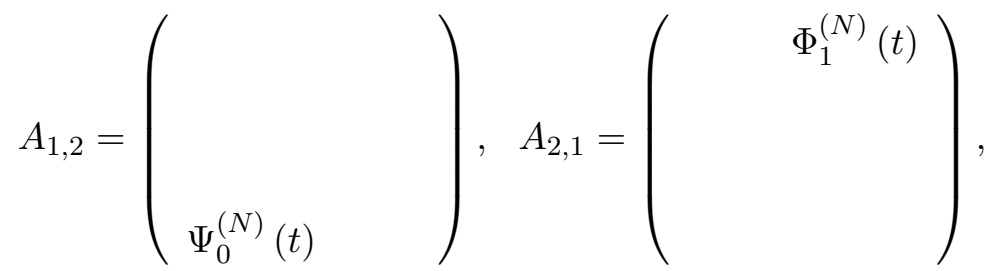

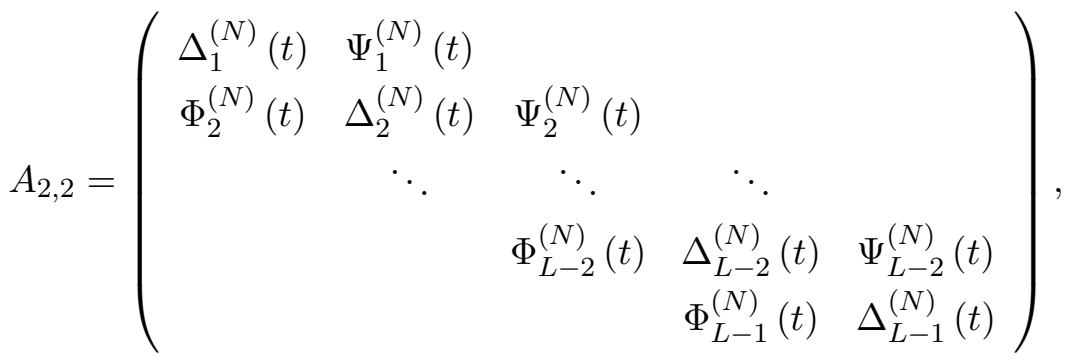

$$
\begin{aligned}
& A_{2,3}=\left(\begin{array}{l}
\Phi_{C}^{(N)}(t) \\
\Psi_{L-1}^{(N)}(t)
\end{array}\right), A_{3,2}=\left(\begin{array}{l}
{ } \\
\end{array}\right), \\
& A_{3,3}=\left(\begin{array}{ccccc}
\Delta_{C}^{(N)}(t) & \Psi_{C}^{(N)}(t) & & & \\
\Phi_{C+1}^{(N)}(t) & \Delta_{C+1}^{(N)}(t) & \Psi_{C+1}^{(N)}(t) & & \\
& \ddots & \ddots & \ddots & \\
& & \Phi_{K-2}^{(N)}(t) & \Delta_{K-2}^{(N)}(t) & \Psi_{K-2}^{(N)}(t) \\
& & & \Phi_{K-1}^{(N)}(t) & \Delta_{K-1}^{(N)}(t)
\end{array}\right), \\
& A_{3,4}=\left(\begin{array}{l}
\Phi_{K}^{(N)}(t) \\
\Psi_{K-1}^{(N)}(t)
\end{array}\right), A_{4,3}=\left(\begin{array}{l} 
\\
\end{array}\right), \\
& A_{4,4}=\left(\begin{array}{ccccc}
\Delta_{K}^{(N)}(t) & \Psi_{K}^{(N)}(t) & & & \\
\Phi_{K+1}^{(N)}(t) & \Delta_{K+1}^{(N)}(t) & \Psi_{K+1}^{(N)}(t) & & \\
& \ddots & \ddots & \ddots & \\
& & \Phi_{K+L-1}^{(N)}(t) & \Delta_{K+L-1}^{(N)}(t) & \Psi_{K+L-1}^{(N)}(t) \\
& & & \Phi_{K+L}^{(N)}(t) & \widehat{\Phi}_{K+L}^{(N)}(t)
\end{array}\right) .
\end{aligned}
$$

Remark 4 To set up the block-structured system of mean-field equations, it is a key to observe two figures: Figure 1 shows all the original parameters of the queueing process under Markovian environment when one isolated station is paid attention to. Figure 4 
further gives mean-field expressions for the transition rates of the $Q B D$ process between two neighboring levels by considering the weak interactions among the $N$ stations of the bike sharing system in terms of the mean-field theory.

\section{The Martingale Limits}

In this section, we apply the martingale limit theory to prove the asymptotic independence of this bike sharing system, that is, the sequence $\left\{\mathbf{Y}^{(N)}(t), t \geq 0\right\}$ of Markov processes asymptotically approaches a single trajectory identified by a solution to the block-structured system of limiting mean-field equations.

For the vector $\mathbf{g}=\left(g_{-L}, g_{-(L-1)}, \ldots, g_{K+L-1}, g_{K+L}\right)$ where $g_{k}=\left(g_{k, 1}, g_{k, 2}, \ldots, g_{k, m}\right)$, we set

$$
\Omega_{N}=\{\mathbf{g}: \mathbf{g} \geq 0, \mathbf{g} e=1, N \mathbf{g} \text { is a vector of nonnegative integers }\}
$$

and

$$
\Omega=\{\mathbf{g}: \mathbf{g} \geq 0, \mathbf{g} e=1\} .
$$

Obviously, $\Omega_{N} \subset \Omega$. In the vector space $\Omega\left(\right.$ or $\Omega_{N}$ ), we take a metric

$$
\rho\left(\mathbf{g}, \mathbf{g}^{\prime}\right)=\max _{-L \leq k \leq K+L} \max _{1 \leq j \leq m}\left\{\left|g_{k, j}-g_{k, j}^{\prime}\right|\right\}, \quad \mathbf{g}, \mathbf{g}^{\prime} \in \Omega .
$$

Note that under the metric $\rho\left(\mathbf{g}, \mathbf{g}^{\prime}\right)$, the vector space $\Omega\left(\right.$ or $\Omega_{N}$ ) is separable and compact.

Now we consider the Markov process $\left\{\mathbf{Y}^{(N)}(t), t \geq 0\right\}$ on state space $\Omega_{N}$ for $N=$ $1,2,3, \ldots$. Note that the stochastic evolution of this bike sharing system is described as the Markov process $\left\{\mathbf{Y}^{(N)}(t), t \geq 0\right\}$, and

$$
\frac{\mathrm{d}}{\mathrm{d} t} \mathbf{Y}^{(N)}(t)=\mathbf{A}_{N} f\left(\mathbf{Y}^{(N)}(t)\right)
$$

where $\mathbf{A}_{N}$ acting on functions $f: \Omega_{N} \rightarrow \mathbf{C}^{1}$ is the generating operator of the Markov process $\left\{\mathbf{Y}^{(N)}(t), t \geq 0\right\}$, and

$$
\mathbf{A}_{N}=\mathbf{A}_{N}^{\text {renting }}+\mathbf{A}_{N}^{\text {returning }}+\mathbf{A}_{N}^{\text {environment }}
$$

where

$$
\begin{aligned}
\mathbf{A}_{N}^{\text {renting }} f(\mathbf{g})= & N \sum_{j=1}^{m} \lambda_{j} \sum_{k=1}^{K+L} g_{k, j}\left[f\left(\mathbf{g}-\frac{e_{k, j}}{N}\right)-f(\mathbf{g})\right] \\
& +N \alpha \sum_{j=1}^{m} \lambda_{j} \sum_{k=-(L-1)}^{0} g_{k, j}\left[f\left(\mathbf{g}-\frac{e_{k, j}}{N}\right)-f(\mathbf{g})\right],
\end{aligned}
$$




$$
\begin{aligned}
\mathbf{A}_{N}^{\text {environment }} & f(\mathbf{g})=N \sum_{i=1}^{m} \sum_{j=1}^{m} \sum_{k=-L}^{K+L} g_{k, i} w_{i, j}\left[f\left(\mathbf{g}-\frac{e_{k, i}}{N}+\frac{e_{k, j}}{N}\right)-f(\mathbf{g})\right], \\
\mathbf{A}_{N}^{\text {returning }} f(\mathbf{g})= & \left\{\sum_{j=1}^{m} \sum_{l=-L}^{0} \mu_{j} g_{l, j} \Theta^{(N)}(0)+\sum_{j=1}^{m} \sum_{l=1}^{C-1} \mu_{j} g_{l, j} \Theta^{(N)}(l)\right. \\
& \left.+\sum_{j=1}^{m} \sum_{l=C}^{K-1} \mu_{j} g_{l, j} \Theta^{(N)}(C)+\beta \sum_{j=1}^{m} \sum_{l=K}^{K+L-1} \mu_{j} g_{l, j} \Theta^{(N)}(C)\right\}\left[f\left(\mathbf{g}+\frac{e_{k, j}}{N}\right)-f(\mathbf{g})\right],
\end{aligned}
$$

and for $0 \leq l \leq C$

$$
\Theta^{(N)}(l)=\left\{C-l+(N-1)\left[C-\sum_{k=1}^{K+L} k g_{k, j}+\sum_{k=K}^{K+L-1} \frac{(1-\beta) g_{k, j}}{\left[1-(1-\beta) g_{k, j}\right]^{2}}+\frac{g_{K+L, j}}{\left[1-g_{K+L, j}\right]^{2}}\right]\right\} .
$$

When $N \rightarrow \infty$, it is easy to check that

$$
\begin{gathered}
N\left[f\left(\mathbf{g}+\frac{e_{k, j}}{N}\right)-f(\mathbf{g})\right] \rightarrow \frac{\partial}{\partial g_{k, j}} f(\mathbf{g}), \\
N\left[f\left(\mathbf{g}-\frac{e_{k, j}}{N}\right)-f(\mathbf{g})\right] \rightarrow-\frac{\partial}{\partial g_{k, j}} f(\mathbf{g}), \\
{\left[f\left(\mathbf{g}-\frac{e_{k, i}}{N}+\frac{e_{k, j}}{N}\right)-f(\mathbf{g})\right] \rightarrow-\frac{\partial}{\partial g_{k, i}} f(\mathbf{g})+\frac{\partial}{\partial g_{k, j}} f(\mathbf{g}),}
\end{gathered}
$$

and for $0 \leq l \leq C$

$$
\begin{aligned}
& \frac{1}{N} \Theta^{(N)}(l)=\frac{1}{N}\left\{C-l+(N-1)\left[C-\sum_{k=1}^{K+L} k g_{k, j}+\sum_{k=K}^{K+L-1} \frac{(1-\beta) g_{k, j}}{\left[1-(1-\beta) g_{k, j}\right]^{2}}+\frac{g_{K+L, j}}{\left[1-g_{K+L, j}\right]^{2}}\right]\right\} \\
& \rightarrow C-\sum_{k=1}^{K+L} k g_{k, j}+\sum_{k=K}^{K+L-1} \frac{(1-\beta) g_{k, j}}{\left[1-(1-\beta) g_{k, j}\right]^{2}}+\frac{g_{K+L, j}}{\left[1-g_{K+L, j}\right]^{2}} \stackrel{\text { Def }}{=} \mathbf{} .
\end{aligned}
$$

Let

$$
\begin{gathered}
\mathbf{A}=\lim _{N \rightarrow \infty} \mathbf{A}_{N}, \quad \mathbf{A}^{\text {renting }}=\lim _{N \rightarrow \infty} \mathbf{A}_{N}^{\text {renting }} \\
\mathbf{A}^{\text {returning }}=\lim _{N \rightarrow \infty} \mathbf{A}_{N}^{\text {returning }}, \quad \mathbf{A}^{\text {environment }}=\lim _{N \rightarrow \infty} \mathbf{A}_{N}^{\text {environment }} .
\end{gathered}
$$

Then

$$
\begin{aligned}
\mathbf{A} f(\mathbf{g})= & -\sum_{j=1}^{m} \lambda_{j} \sum_{k=1}^{K+L} g_{k, j} \frac{\partial}{\partial g_{k, j}} f(\mathbf{g})-\alpha \sum_{j=1}^{m} \lambda_{j} \sum_{k=-(L-1)}^{0} g_{k, j} \frac{\partial}{\partial g_{k, j}} f(\mathbf{g}) \\
& +\sum_{i=1}^{m} \sum_{j=1}^{m} \sum_{k=-L}^{K+L} g_{k, i} w_{i, j}\left[-\frac{\partial}{\partial g_{k, i}} f(\mathbf{g})+\frac{\partial}{\partial g_{k, j}} f(\mathbf{g})\right] \\
& +\boldsymbol{\Theta}\left(\sum_{j=1}^{m} \sum_{l=-L}^{K-1} \mu_{j} g_{l, j}+\beta \sum_{j=1}^{m} \sum_{l=K}^{K+L-1} \mu_{j} g_{l, j}\right) \frac{\partial}{\partial g_{k, j}} f(\mathbf{g}) .
\end{aligned}
$$


Now, we discuss the weak convergence of the sequence $\left\{\mathbf{Y}^{(N)}(t): t \geq 0\right\}$ of Markov processes for $N=1,2,3, \ldots$ Here, our main purpose is to provide some basic support for our later study of various convergence involved. To this end, we consider the random vector $\mathbf{Y}^{(N)}(t)$ with samples in $\mathcal{P}\left(\mathbb{D}\left(R_{+}, \mathbf{N}\right)\right)$, where $R_{+}=[0,+\infty), \mathbf{N}=$ $((k, j):-L \leq k \leq K+L, 1 \leq j \leq m), \mathbb{D}\left(R_{+}, \mathbf{N}\right)$ is the Skorohod space, i.e., the set of mappings which are right continuous with left-hand limits (in short, Càdlàg), and $\mathcal{P}(\cdot)$ is the set of probability measures defined in $\mathbb{D}\left(R_{+}, \mathbf{N}\right)$. Notice that the convergence in the Skorohod topology means the convergence in distribution (or weak convergence) for the Skorohod topology on the space of trajectories. When the sequence $\left\{\mathbf{Y}^{(N)}(t), t \geq 0\right\}$ of Markov processes converges in probability (or converges weakly), for the Skorohod topology, to a given probability vector $\mathbf{Y}(t)$, we write the weak convergence as $\mathbf{Y}^{(N)}(t) \Longrightarrow$ $\mathbf{Y}(t)$ for $t \geq 0$, as $N \longrightarrow \infty$.

If $\mathbf{Y}^{(N)}(t) \Longrightarrow \mathbf{Y}(t)$ for $t \geq 0$ as $N \longrightarrow \infty$, then it is easy to see from (12) and (13) that the transition probabilities of the Markov process $\left\{\mathbf{Y}^{(N)}(t), t \geq 0\right\}$ with generating operator $\mathbf{A}_{N}$ uniformly converges on any finite time interval to the transition probabilities of the limiting Markov process $\{\mathbf{Y}(t), t \geq 0\}$ with generating operator $\mathbf{A}$.

Now we consider the limiting behavior of the sequence $\left\{\mathbf{Y}^{(N)}(t), t \geq 0\right\}$ of Markov processes as $N \rightarrow \infty$. To that end, we first give a system of limiting mean-field equations (14) to (15) below.

Set

$$
\mathbf{y}(t)=\lim _{N \rightarrow \infty} \mathbf{y}^{(N)}(t)
$$

and

$$
\mathbf{V}_{\mathbf{y}(t)}=\lim _{N \rightarrow \infty} \mathbf{V}_{\mathbf{y}^{(N)}(t)} .
$$

Then it follows from (9) and (10) that

$$
\begin{gathered}
\frac{\mathrm{d}}{\mathrm{d} t} \mathbf{y}(t)=\mathbf{y}(t) \mathbf{V}_{\mathbf{y}(t)}, \\
\mathbf{y}(t) e=1, \mathbf{y}(0)=\mathbf{g} \in \boldsymbol{\Omega} .
\end{gathered}
$$

Note that the convergence in the Skorohod topology means the convergence in distribution for the Skorohod topology on the space of trajectories. The following theorem applies the martingale limit theory to discuss the weak convergence of the sequence $\left\{\mathbf{Y}^{(N)}(t), t \geq 0\right\}$ of Markov processes as $N$ tends to infinity. 
Theorem 2 If $\mathbf{Y}^{(N)}(0)$ converges weakly to $\mathbf{g} \in \Omega$ as $N$ tends to infinity, then the sequence $\left\{\mathbf{Y}^{(N)}(t), t \geq 0\right\}$ of Markov processes converges in the Skorohod topology to a solution $\mathbf{y}(t)$ to the system of limiting mean-field equations (14) to (15).

Proof: From the martingale characterization of the Markov jump process $\left\{\mathbf{Y}^{(N)}(t), t \geq 0\right\}$, it follows from Rogers and Williams [39, 38] that for $-L \leq k \leq K+L$ and $1 \leq j \leq m$,

$M_{k, j}^{(N)}(t)=Y_{k, j}^{(N)}(t)-Y_{k, j}^{(N)}(0)-\int_{0}^{t} \sum_{\Lambda \in \Omega-\left\{\mathbf{Y}^{(N)}(t)\right\}} \mathcal{Q}^{(N)}\left(\mathbf{Y}^{(N)}(s), \Lambda\right)\left[\Lambda_{k, j}-Y_{k, j}^{(N)}(s)\right] \mathrm{d} s$ is a martingale with respect to the natural filtration associated to the Poisson processes involved in the renting and returning processes and to the Markov process of the Markovian environment, where $\mathcal{Q}^{(N)}\left(\mathbf{Y}^{(N)}(s), \Lambda\right)$ is the $Q$-matrix of the Markov jump process $\left\{\mathbf{Y}^{(N)}(t), t \geq 0\right\}$ whose expression is given by means of the state change due to the renting and returning processes as well as the state transitions of the Markovian environment.

To express the $Q$-matrix $\mathcal{Q}^{(N)}\left(\mathbf{Y}^{(N)}(s), \Lambda\right)$, we analyze three classes of state transitions as follows:

(1) When a customer arrives at the tagged station to rent a bike, the state transition rate is given by

$$
q_{k, j ; k-1, j}= \begin{cases}\lambda_{j}, \quad 1 \leq k \leq K+L, & 1 \leq j \leq m \\ \lambda_{j} \alpha, \quad-(L-1) \leq k \leq 0, & 1 \leq j \leq m\end{cases}
$$

(2) When a customer returns his bike to the tagged station, the state transition rate 
is given by

$$
q_{k, j ; k+1, j}^{(N)}(t)=\left\{\begin{array}{l}
\frac{\mu_{j}}{N}\left\{C+(N-1)\left[C-\sum_{k=1}^{K+L} k y_{k, j}^{(N)}(t)\right.\right. \\
\left.\left.+\sum_{k=K}^{K+L-1} \frac{(1-\beta) y_{k, j}^{(N)}(t)}{\left[1-(1-\beta) y_{k, j}^{(N)}(t)\right]^{2}}+\frac{y_{K+L, j}^{(N)}(t)}{\left[1-y_{K+L, j}^{(N)}(t)\right]^{2}}\right]\right\}, \quad-L \leq l \leq 0, \\
\frac{\mu_{j}}{N}\left\{C-l+(N-1)\left[C-\sum_{k=1}^{K+L} k y_{k, j}^{(N)}(t)\right.\right. \\
\left.\left.+\sum_{k=1}^{K+L-1} \frac{(1-\beta) y_{k, j}^{(N)}(t)}{\left[1-(1-\beta) y_{k, j}^{(N)}(t)\right]^{2}}+\frac{y_{K+L, j}^{(N)}(t)}{\left[1-y_{K+L, j}^{(N)}(t)\right]^{2}}\right]\right\}, \quad 1 \leq l \leq C-1, \\
\frac{\mu_{j}}{N}\left\{( N - 1 ) \left[C-\sum_{k=1}^{K+L} k y_{k, j}^{(N)}(t)\right.\right. \\
\left.\left.+\sum_{k=1}^{K+L-1} \frac{(1-\beta) y_{k, j}^{(N)}(t)}{\left[1-(1-\beta) y_{k, j}^{(N)}(t)\right]^{2}}+\frac{y_{K+L, j}^{(N)}(t)}{\left[1-y_{K+L, j}^{(N)}(t)\right]^{2}}\right]\right\}, \quad C \leq l \leq K-1, \\
\beta \frac{\mu_{j}}{N}\left\{( N - 1 ) \left[C-\sum_{k=1}^{K+L} k y_{k, j}^{(N)}(t)\right.\right. \\
\left.\left.+\sum_{k=1}^{K+L-1} \frac{(1-\beta) y_{k, j}^{(N)}(t)}{\left[1-(1-\beta) y_{k, j}^{(N)}(t)\right]^{2}}+\frac{y_{K+L, j}^{(N)}(t)}{\left[1-y_{K+L, j}^{(N)}(t)\right]^{2}}\right]\right\}, \quad K \leq l \leq K+L-1 .
\end{array}\right.
$$

(3) When the Markovian environment changes from State $i$ to State $j$, the state transition rate is given by

$$
q_{k, i ; k, j}=w_{i, j}, \quad-L \leq k \leq K+L, i \neq j, 1 \leq i, j \leq m
$$

Based on the above three cases, the $Q$-matrix $\mathcal{Q}^{(N)}\left(\mathbf{Y}^{(N)}(s), \Lambda\right)$ is given by

$$
\begin{aligned}
Y_{k, j}^{(N)}(t)= & M_{k, j}^{(N)}(t)+Y_{k, j}^{(N)}(0)+q_{k+1, j ; k, j} \int_{0}^{t} Y_{k+1, j}^{(N)}(s) \mathrm{d} s \\
& +\int_{0}^{t} q_{k-1, j ; k, j}^{(N)}(s) Y_{k-1, j}^{(N)}(s) \mathrm{d} s+\sum_{i \neq j}^{m} w_{i, j} \int_{0}^{t} Y_{k, i}^{(N)}(s) \mathrm{d} s
\end{aligned}
$$

Using a similar method to Darling and Norris [4, 5], it is easy to see that if $\mathbf{Y}^{(N)}(0)$ converges weakly to $\mathbf{g} \in \Omega$ as $N$ tends to infinity, then the sequence $\left\{\mathbf{Y}^{(N)}(t), t \geq 0\right\}$ of Markov processes is tight for the Skorohod topology, and any limit $\mathbf{Y}(t)$ of $\left\{\mathbf{Y}^{(N)}(t), t \geq 0\right\}$ asymptotically approaches to a single trajectory identified by a solution $\mathbf{y}(t)$ to the system of limiting mean-field equations (14) to (15). This completes the proof.

\section{A Nonlinear QBD Process}

In this section, we discuss the fixed point of the block-structured system of limiting meanfield equations (14) to (15), and provide a mean-field matrix-analytic method which can 
be used to numerically compute the fixed point. Furthermore, we study the limiting interchangeability of $\mathbf{y}^{(N)}(t)$ as $N \rightarrow \infty$ and $t \rightarrow+\infty$, that is, the asymptotic independency, which plays a key role in approximate computation for performance measures of this bike sharing system.

We rewrite the system of limiting mean-field equations (14) to (15) as

$$
\frac{\mathrm{d}}{\mathrm{d} t} \mathbf{y}(t)=\mathbf{y}(t) \mathbf{V}_{\mathbf{y}(t)}
$$

and

$$
\mathbf{y}(t) e=1, \mathbf{y}(0)=\mathbf{g} \in \mathbf{\Omega} .
$$

A point $\pi \in \Omega$ is said to be a fixed point if $\lim _{t \rightarrow+\infty}\left[\frac{\mathrm{d}}{\mathrm{d} t} \mathbf{y}(t)\right]=0$, or

$$
\left[\mathbf{y}(t) \mathbf{V}_{\mathbf{y}(t)}\right]_{\mid \mathbf{y}(t)=\pi}=0 \text {. }
$$

Thus, we have

$$
\pi \mathbf{V}_{\pi}=0
$$

and

$$
\pi e=1
$$

Now, we provide a mean-field matrix-analytic method to compute the fixed point $\pi$ from the system of nonlinear equations: $\pi \mathbf{V}_{\pi}=0$ and $\pi \mathbf{e}=1$. To this end, it is necessary to explore the block structure of the system of nonlinear equations. Hence this gives a nonlinear QBD process so that the $R G$-factorizations given by $\mathrm{Li}$ [21] are applicable in our later analysis.

Let

$$
\xi_{k, j}=\lim _{t \rightarrow+\infty} \lim _{N \rightarrow \infty} \xi_{k, j}^{(N)}(t), \quad-L \leq k \leq K+L, 1 \leq j \leq m .
$$

Then

$$
\xi_{k, j}=\left\{\begin{array}{l}
\mu_{j} \zeta_{j}, \quad-L \leq k \leq K-1, \quad 1 \leq j \leq m \\
\beta \mu_{j} \zeta_{j}, \quad K \leq k \leq K+L-1, \quad 1 \leq j \leq m
\end{array}\right.
$$

where

$$
\zeta_{j}=C-\sum_{k=1}^{K+L} k \pi_{k, j}+\sum_{k=K}^{K+L-1} \frac{(1-\beta) \pi_{k, j}}{\left[1-(1-\beta) \pi_{k, j}\right]^{2}}+\frac{\pi_{K+L, j}}{\left[1-\pi_{K+L, j}\right]^{2}} .
$$


Table 1: The special structures of the four functions $\Psi_{k}, \widehat{\Psi}_{k}, \Phi_{k}$ and $\widehat{\Phi}_{k}$

\begin{tabular}{|c|c|c|c|c|c|}
\hline$K$ & $-L$ & $-L+1 \leq k \leq 0$ & $1 \leq k \leq K-1$ & $K \leq k \leq K+L-1$ & $K+L$ \\
\hline$\Psi_{k}$ & $\Psi(1)$ & $\Psi(1)$ & $\Psi(1)$ & $\Psi(\beta)$ & null \\
\hline$\widehat{\Psi}_{k}$ & $\widehat{\Psi}(1)$ & $\widehat{\Psi}(1)$ & $\widehat{\Psi}(1)$ & $\widehat{\Psi}(\beta)$ & null \\
\hline$\Phi_{k}$ & null & $\Phi(\alpha)$ & $\Phi(1)$ & $\Phi(1)$ & $\Phi(1)$ \\
\hline$\widehat{\Phi}_{k}$ & null & $\widehat{\Phi}(\alpha)$ & $\widehat{\Phi}(1)$ & $\widehat{\Phi}(1)$ & $\widehat{\Phi}(1)$ \\
\hline
\end{tabular}

Thus for $K \leq k \leq K+L-1$,

$$
\begin{gathered}
\Psi_{k}=\left(\begin{array}{cccc}
0 & \beta \mu_{1} \zeta_{1} w_{1, m} & \cdots & \beta \mu_{1} \zeta_{1} w_{1, m} \\
\beta \mu_{2} \zeta_{2} w_{2,1} & 0 & \cdots & \beta \mu_{2} \zeta_{2} w_{2, m} \\
\vdots & \vdots & \ddots & \vdots \\
\beta \mu_{m} \zeta_{m} w_{m, 1} & \beta \mu_{m} \zeta_{m} w_{m, 2} & \cdots & 0
\end{array}\right) \stackrel{\text { Def }}{=} \Psi(\beta), \\
\widehat{\Psi}_{k}=\operatorname{diag}\left(\beta \mu_{1} \zeta_{1} w_{1,1}, \beta \mu_{2} \zeta_{2} w_{2,2}, \ldots, \beta \mu_{m} \zeta_{m} w_{m, m}\right) \stackrel{\text { Def }}{=} \widehat{\Psi}(\beta) ;
\end{gathered}
$$

and for $-L \leq k \leq K-1$,

$$
\Psi_{k}=\Psi(1)
$$

and

$$
\widehat{\Psi}_{k}=\widehat{\Psi}(1)
$$

Based on the above analysis, we can summarize the special structures of the four functions $\Psi_{k}, \widehat{\Psi}_{k}, \Phi_{k}$ and $\widehat{\Phi}_{k}$ in Table 1 .

By observing Table 1, it is easy to check from (11) that as $N \rightarrow \infty$ and $t \rightarrow+\infty$

$$
\mathbf{V}_{\pi}=\left(\begin{array}{ccc}
B_{1,1} & B_{1,2} & \\
B_{2,1} & B_{2,2} & B_{2,3} \\
& B_{3,2} & B_{3,3}
\end{array}\right)
$$

where

$$
B_{1,1}=\left(\begin{array}{ccccc}
\widehat{\Psi}_{-L} & \Psi_{-L} & & & \\
\Phi_{-(L-1)} & \widehat{\Phi}_{-(L-1)}+\widehat{\Psi}_{-(L-1)} & \Psi_{-(L-1)} & & \\
& \ddots & \ddots & \ddots & \\
& & \Phi_{-1} & \widehat{\Phi}_{-1}+\widehat{\Psi}_{-1} & \Psi_{-1} \\
& & & \Phi_{0} & \widehat{\Phi}_{0}+\widehat{\Psi}_{0}
\end{array}\right)
$$




$$
\begin{aligned}
& B_{1,2}=\left(\begin{array}{l} 
\\
\Psi_{0}
\end{array}\right), \quad B_{2,1}=\left(\begin{array}{l}
\Phi_{1} \\
\end{array}\right), \\
& B_{2,2}=\left(\begin{array}{ccccc}
\widehat{\Phi}_{1}+\widehat{\Psi}_{1} & \Psi_{1} & & & \\
\Phi_{2} & \widehat{\Phi}_{2}+\widehat{\Psi}_{2} & \Psi_{2} & & \\
& \ddots & \ddots & \ddots & \\
& & \Phi_{K-2} & \widehat{\Phi}_{K-2}+\widehat{\Psi}_{K-2} & \Psi_{K-2} \\
& & & \Phi_{K-1} & \widehat{\Phi}_{K-1}+\widehat{\Psi}_{K-1}
\end{array}\right) \text {, }
\end{aligned}
$$

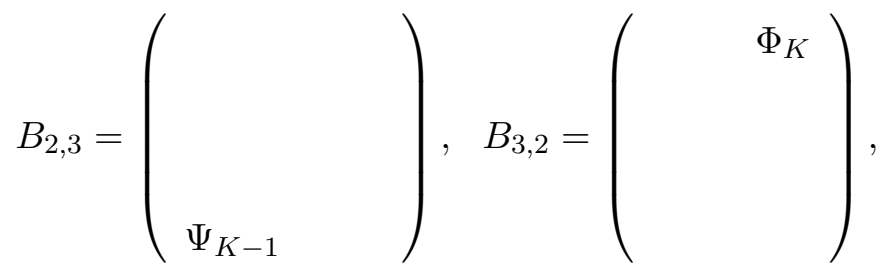

$$
\begin{aligned}
& B_{3,3}=\left(\begin{array}{ccccc}
\widehat{\Phi}_{K}+\widehat{\Psi}_{K} & \Psi_{K} & & & \\
\Phi_{K+1} & \widehat{\Phi}_{K+1}+\widehat{\Psi}_{K+1} & \Psi_{K+1} & & \\
& \ddots & \ddots & \ddots & \\
& & \Phi_{K+L-1} & \widehat{\Phi}_{K+L-1}+\widehat{\Psi}_{K+L-1} & \Psi_{K+L-1} \\
& & & \Phi_{K+L} & \widehat{\Phi}_{K+L}
\end{array}\right) .
\end{aligned}
$$

Thus it follows from (18) that

$$
\mathbf{V}_{\pi}=\left(\begin{array}{ccccc}
\widehat{\Psi}_{-L} & \Psi_{-L} & & & \\
\Phi_{-(L-1)} & \widehat{\Phi}_{-(L-1)}+\widehat{\Psi}_{-(L-1)} & \Psi_{-(L-1)} & & \\
& \ddots & \ddots & \ddots & \\
& & \Phi_{K+L-1} & \widehat{\Phi}_{K+L-1}+\widehat{\Psi}_{K+L-1} & \Psi_{K+L-1} \\
& & & \Phi_{K+L} & \widehat{\Phi}_{K+L}
\end{array}\right) .
$$

Based on the nonlinear QBD process $\mathbf{V}_{\pi}$, we write

$$
\pi=\left(\pi_{-L}, \pi_{-L+1}, \ldots, \pi_{K+L-1}, \pi_{K+L}\right) .
$$

Now, we use the LU-type $R G$-factorization given in Subsection 1.3.2 of Chapter one in Li [21] (see Pages 25 and 26), and write the LU-type R-measure as

$$
\mathbf{R}_{-L+1}(\pi)=-\Phi_{-L+1}\left(\widehat{\Psi}_{-L}\right)^{-1}
$$




$$
\mathbf{R}_{-L+2}(\pi)=-\Phi_{-L+2}\left[\mathbf{R}_{-L+1}(\pi) \Psi_{-L}+\left(\widehat{\Phi}_{-L+1}+\widehat{\Psi}_{-L+1}\right)\right]^{-1}
$$

for $-L+2 \leq k \leq K+L$,

$$
\mathbf{R}_{k}(\pi)=-\Phi_{k}\left[\mathbf{R}_{k-1}(\pi) \Psi_{k-2}+\left(\widehat{\Phi}_{k-1}+\widehat{\Psi}_{k-1}\right)\right]^{-1} .
$$

At the same time, the infinitesimal generator of the censored Markov chain to level $K+L$ is given by

$$
\boldsymbol{\Xi}_{K+L}=\mathbf{R}_{K+L}(\pi) \Psi_{K+L-1}+\widehat{\Phi}_{K+L}
$$

For the fixed point $\pi=\left(\pi_{-L}, \pi_{-L+1}, \ldots, \pi_{K+L-1}, \pi_{K+L}\right)$, it follows from (1.25) in Subsection 1.3.4.1 of Li [21] (see Page 30) that for $k=-L$,

$$
\pi_{-L}=\pi_{-L+1} \mathbf{R}_{-L+1}(\pi),
$$

and for $-L+1 \leq k \leq K+L-1$,

$$
\begin{aligned}
\pi_{k} & =\pi_{k+1} \mathbf{R}_{k+1}(\pi) \\
& =\pi_{K+L} \mathbf{R}_{K+L}(\pi) \mathbf{R}_{K+L-1}(\pi) \mathbf{R}_{K+L-2}(\pi) \cdots \mathbf{R}_{k+1}(\pi),
\end{aligned}
$$

where the vector $\pi_{K+L}$ is a solution to the systems of nonlinear equations $\pi_{K+L} \boldsymbol{\Xi}_{K+L}=0$ and $\pi_{K+L}\left[I+\sum_{k=-L}^{K+L-1} \mathbf{R}_{K+L}(\pi) \mathbf{R}_{K+L-1}(\pi) \mathbf{R}_{K+L-2}(\pi) \cdots \mathbf{R}_{k+1}(\pi)\right] e=1$.

The following theorem is a summarization of the above analysis, and its proof is easy to only check the system of nonlinear equations $\pi \mathbf{V}_{\pi}=0$ and $\pi e=1$. Thus we omit the proof here.

Theorem 3 The fixed point $\pi$ is a solution to the vector system of nonlinear equations

$$
\begin{aligned}
\pi= & \left(\pi_{K+L} \mathbf{R}_{K+L}(\pi) \mathbf{R}_{K+L-1}(\pi) \mathbf{R}_{K+L-2}(\pi) \cdots \mathbf{R}_{-L+1}(\pi),\right. \\
& \pi_{K+L} \mathbf{R}_{K+L}(\pi) \mathbf{R}_{K+L-1}(\pi) \mathbf{R}_{K+L-2}(\pi) \cdots \mathbf{R}_{-L+2}(\pi), \\
& \left.\ldots, \pi_{K+L} \mathbf{R}_{K+L}(\pi) \mathbf{R}_{K+L-1}(\pi), \pi_{K+L} \mathbf{R}_{K+L}(\pi), \pi_{K+L}\right), \\
& \pi_{K+L}\left[\mathbf{R}_{K+L}(\pi) \Psi_{K+L-1}+\left(\widehat{\Phi}_{K+L}+\widehat{\Psi}_{K+L}\right)\right]=0
\end{aligned}
$$

and

$$
\pi_{K+L}\left[I+\sum_{k=-L}^{K+L-1} \mathbf{R}_{K+L}(\pi) \mathbf{R}_{K+L-1}(\pi) \mathbf{R}_{K+L-2}(\pi) \cdots \mathbf{R}_{k+1}(\pi)\right] e=1 .
$$


It is easy to see that although the system of nonlinear equations: $\pi \mathbf{V}_{\pi}=0$ and $\pi e=1$, are equivalent to the vector system of nonlinear equations (19), (20) and (21), Theorem 3 can be used to design different algorithms to numerically compute the fixed points $\pi$. This is indicated in the next section with six numerical examples. Reader may also refer to $\mathrm{Li}$ [22] and Li et al. 28] for some nearby research.

In what follows we discuss the mean-field limit of the empirical measure process of the bike sharing system as the number $N$ of stations and time $t$ go to infinity, and show that the fixed point is unique from the system of nonlinear equations: $\pi \mathbf{V}_{\pi}=0$ and $\pi e=1$. It is worthwhile to note that the uniqueness of the fixed point guarantees the asymptotic independence of the queueing processes describing the numbers of bikes at the $N$ stations as $N \rightarrow \infty$, also known as the propagation of chaos.

For the unique fixed point $\pi$, we discuss the limiting interchangeability of the probability vector $\mathbf{y}^{(N)}(t, \mathbf{g})$ as $N \rightarrow \infty$ and $t \rightarrow+\infty$, where $\mathbf{y}^{(N)}(0, \mathbf{g})=\mathbf{g} \in \boldsymbol{\Omega}$. Note that the limiting interchangeability is necessary in many practical applications when using the stationary probabilities (that is, the fixed point) of the limiting process to give an effective approximation for performance analysis of this bike sharing system.

The following theorem gives the limit of the vector $\mathbf{y}(t, \mathbf{g})$ as $t \rightarrow+\infty$, that is,

$$
\mathbf{y}(t, \mathbf{g})=\lim _{N \rightarrow \infty} \mathbf{y}^{(N)}(t, \mathbf{g})
$$

and

$$
\lim _{t \rightarrow+\infty} \mathbf{y}(t, \mathbf{g})=\lim _{t \rightarrow+\infty} \lim _{N \rightarrow \infty} \mathbf{y}^{(N)}(t, \mathbf{g})
$$

Theorem 4 For any $\mathrm{g} \in \Omega$

$$
\lim _{t \rightarrow+\infty} \mathbf{y}(t, \mathbf{g})=\pi
$$

Furthermore, there exists a unique probability measure $\varphi$ on $\Omega$, which is invariant under the map $\mathbf{g} \longmapsto \mathbf{y}(t, \mathbf{g})$, that is, for any continuous function $f: \Omega \rightarrow \mathbf{R}$ and $t>0$

$$
\int_{\Omega} f(\mathbf{g}) d \varphi(\mathbf{g})=\int_{\Omega} f(\mathbf{y}(t, \mathbf{g})) d \varphi(\mathbf{g}) .
$$

Also, $\varphi=\delta_{\pi}$ is the probability measure concentrated at the fixed point $\pi$.

Proof: It is seen from Theorem 2 that as $t \rightarrow+\infty$, the limit of $\mathbf{y}(t, \mathbf{g})$ exists on $\Omega$, and it is also a solution on $\Omega$ to the system of nonlinear equations (16) and (17). Since $\mathbf{y}(t, \mathbf{g})$ is the unique solution to the system of limiting mean-field equations (14) and (15), 
the vector $\lim _{t \rightarrow+\infty} \mathbf{y}(t, \mathbf{g})$ is also a solution to the system of nonlinear equations (16) and (17). Note that $\pi$ is the unique solution to the system of nonlinear equations (16) and (17), hence we obtain that $\lim _{t \rightarrow+\infty} \mathbf{y}(t, \mathbf{g})=\pi$. The second statement in this theorem can be immediately given by the probability measure of the limiting process $\{\mathbf{Y}(t), t \geq 0\}$ on state space $\Omega$. This completes the proof.

The following theorem indicates the weak convergence of the sequence $\left\{\varphi_{N}\right\}$ of stationary probability distributions for the sequence $\left\{\mathbf{Y}^{(N)}(t), t \geq 0\right\}$ of Markov processes to the probability measure concentrated at the fixed point $\pi$.

Theorem 5 (1) For a fixed number $N=1,2,3, \ldots$, the Markov process $\left\{\mathbf{Y}^{(N)}(t), t \geq 0\right\}$ is positive recurrent, and has a unique invariant distribution $\varphi_{N}$.

(2) $\left\{\varphi_{N}\right\}$ weakly converges to $\delta_{\pi}$, that is, for any continuous function $f: \Omega \rightarrow \mathbf{R}$

$$
\lim _{N \rightarrow \infty} E_{\varphi_{N}}[f(\mathbf{g})]=f(\pi) .
$$

Proof: (1) From Theorem 3, this bike sharing system of $N$ identical stations is stable, hence this bike sharing system has a unique invariant distribution $\varphi_{N}$.

(2) Since $\Omega$ is compact under the metric $\rho\left(\mathbf{g}, \mathbf{g}^{\prime}\right)$, so it is the set $\mathcal{P}(\Omega)$ of probability measures. Hence the sequence $\left\{\varphi_{N}\right\}$ of invariant distributions has limiting points. A similar analysis to the proof of Theorem 5 in Martin and Suhov [31] shows that $\left\{\varphi_{N}\right\}$ weakly converges to $\delta_{\pi}$ and $\lim _{N \rightarrow \infty} E_{\varphi_{N}}[f(\mathbf{g})]=f(\pi)$. This completes the proof.

Based on Theorems 4 and 5, we obtain a useful relation as follows

$$
\lim _{t \rightarrow+\infty} \lim _{N \rightarrow \infty} \mathbf{y}^{(N)}(t, \mathbf{g})=\lim _{N \rightarrow \infty} \lim _{t \rightarrow+\infty} \mathbf{y}^{(N)}(t, \mathbf{g})=\pi .
$$

Therefore, we have

$$
\lim _{\substack{N \rightarrow \infty \\ t \rightarrow+\infty}} \mathbf{y}^{(N)}(t, \mathbf{g})=\pi
$$

which justifies the exchangeability of the limits of $N \rightarrow \infty$ and $t \rightarrow+\infty$.

Finally, we further show the asymptotic independence (or propagation of chaos) of the queueing processes of this bike sharing system for each $k=2,3, \ldots, N$ as follows:

$$
\begin{aligned}
& \lim _{t \rightarrow+\infty} \lim _{N \rightarrow \infty} P\left\{X_{1}^{(N)}(t)=n_{1}, J_{1}(t)=j_{1} ; \ldots ; X_{k}^{(N)}(t)=n_{k}, J_{k}(t)=j_{k}\right\} \\
& =\lim _{N \rightarrow \infty} \lim _{t \rightarrow+\infty} P\left\{X_{1}^{(N)}(t)=n_{1}, J_{1}(t)=j_{1} ; \ldots ; X_{k}^{(N)}(t)=n_{k}, J_{k}(t)=j_{k}\right\} \\
& =\prod_{l=1}^{k} \pi_{n_{l}, j_{l}}
\end{aligned}
$$


and

$$
\begin{aligned}
& \lim _{N \rightarrow \infty} \lim _{t \rightarrow+\infty} \frac{1}{t} \int_{0}^{t} \mathbf{1}_{\left\{X_{1}^{(N)}(t)=n_{1}, J_{1}(t)=j_{1} ; \ldots ; X_{k}^{(N)}(t)=n_{k}, J_{k}(t)=j_{k}\right\}} \mathrm{d} t \\
& =\lim _{t \rightarrow+\infty N \rightarrow \infty} \lim _{N \rightarrow} \frac{1}{t} \int_{0}^{t} \mathbf{1}_{\left\{X_{1}^{(N)}(t)=n_{1}, J_{1}(t)=j_{1} ; \ldots ; X_{k}^{(N)}(t)=n_{k}, J_{k}(t)=j_{k}\right\}} \mathrm{d} t \\
& =\prod_{l=1}^{k} \pi_{n_{l}, j_{l}} \quad \text { a.s. }
\end{aligned}
$$

It is obvious that the asymptotic independence needs to hold for each subset of the $N$ same stations. Based on this, it is easy to see that the two types of limits may be used as an approximate computation for performance measures of this bike sharing system, hence this demonstrates the key role played by the asymptotic independence.

\section{Numerical Analysis}

In this section, we first use the fixed point to express interesting performance measures of this bike sharing system, such as, the stationary average number of bikes at the tagged station, the stationary strong-probability of problematic stations, the stationary weakprobability of problematic stations, and impact of the user's finite waiting rooms on system performance. Then we use six numerical examples to demonstrate how the performance measures depend on some key parameters of this bike sharing system. Therefore, this paper provides numerical solution in the study of more general bike sharing systems by means of the nonlinear QBD processes.

\subsection{Performance measures}

Using the fixed point $\pi=\left(\pi_{-L}, \pi_{-L+1}, \ldots, \pi_{0}, \pi_{1}, \ldots, \pi_{K+L-1}, \pi_{K+L}\right)$ where $\pi_{k}=\left(\pi_{k, 1}, \pi_{k, 2}\right.$, $\left.\ldots, \pi_{k, m}\right)$ and $\pi_{k} e=\sum_{j=1}^{m} \pi_{k, j}$, we provide some interesting performance measures of this bike sharing system from a practical point of view as follows:

(1) The stationary average number of bikes parked at the tagged station

$$
E[Q]=\sum_{k=1}^{K+L} k \pi_{k} e .
$$

(2-1) The stationary average number of waiting places used by customers 
who are renting bikes at the tagged station

$$
E\left[N_{1}\right]=\sum_{k=-L}^{-1}(-k) \pi_{k} e .
$$

(2-2) The stationary average number of waiting places used by customers who are returning bikes at the tagged station

$$
E\left[N_{2}\right]=\sum_{k=K+1}^{K+L}(k-K) \pi_{k} e .
$$

(2-3) The maximal stationary average number of waiting places used at tagged station

$$
E[N]=\max \left\{E\left[N_{1}\right], E\left[N_{2}\right]\right\}=\max \left\{\sum_{k=-L}^{-1}(-k) \pi_{k} e, \sum_{k=K+1}^{K+L}(k-K) \pi_{k} e\right\} .
$$

Since $E\left[N_{1}\right]$ and $E\left[N_{2}\right]$ can not exist simultaneously, $E[N]$ is useful for synthetically designing the user's finite waiting rooms of this bike sharing system.

(3) The stationary strong-probability of problematic stations

The strong-probability of problematic stations is a probability that either there is both no bike $(-L \leq k \leq 0)$ and no empty waiting place $(k=-L)$ when renting a bike, or there is both no parking place $(K \leq k \leq K+L)$ and no empty waiting place $(k=K+L)$ when returning a bike. Thus the stationary strong-probability of problematic stations is given by

$$
\mathbf{p}_{s}=\pi_{-L} e+\pi_{K+L} e
$$

We consider the effect of the size of waiting places on the strong-probability of problematic stations. Let

$$
v=\frac{\pi_{-L} e+\pi_{K+L} e}{\left(\pi_{-L} e+\pi_{K+L} e\right)_{\mid L=0}} .
$$

Then $v$ denotes the improved efficiency of problematic stations due to introduction of the user's waiting room of size $L>0$.

\section{(4) The stationary weak-probability of problematic stations}

The weak-probability of problematic stations is the probability that either there is no bike $(-L \leq k \leq 0)$ when renting a bike, or there is no parking place $(K \leq k \leq K+L)$ when returning a bike. Thus the stationary weak-probability of problematic stations is given by

$$
\mathbf{p}_{w}=\sum_{k=-L}^{0} \pi_{k} e+\sum_{k=K}^{K+L} \pi_{k} e
$$



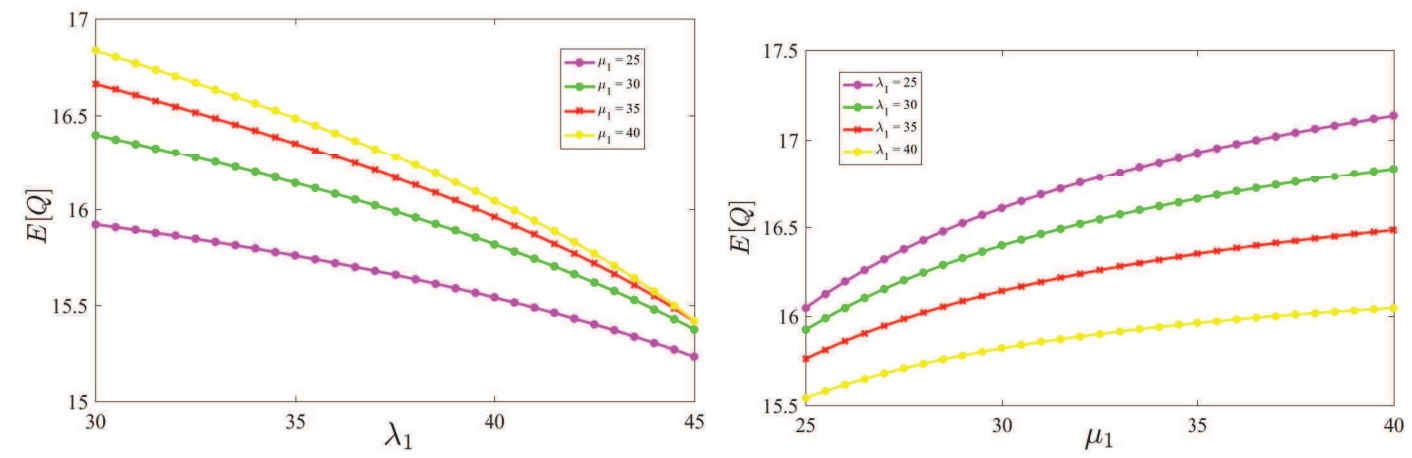

Figure 5: $E[Q]$ vs. $\lambda_{1}$ and $\mu_{1}$

\subsection{Numerical examples}

Now, we use six numerical examples to show how the performance measures depend on some key parameters of this bike sharing system. As illustrated in the following figures, the Markovian environment motivates us to propose the mean-field matrix-analytic method which is necessarily developed as some effective numerical solution in the study of bike sharing systems.

In the following examples one to four, we take some common parameters as follows:

$$
K=20, C=10, L=5, \lambda_{2}=50, \mu_{2}=20, \alpha=0.5, \beta=0.5, m=2, w=\left(\begin{array}{cc}
-1 & 1 \\
1 & -1
\end{array}\right) ;
$$

while the other parameters are conceretely chosen in each example for the target of specific observation.

\section{Example one: Analysis of $E[Q]$}

The left of figure 5 indicates how the stationary average number $E[Q]$ of bikes at the tagged station depends on $\lambda_{1} \in(30,45)$ when $\mu_{1}=25,30,35$ and 40 , respectively. It is seen that $E[Q]$ decreases as $\lambda_{1}$ increases but it increases as $\mu_{1}$ increases. The right of figure 5 shows how $E[Q]$ depends on $\mu_{1} \in(25,40)$ when $\lambda_{1}=25,30,35$ and 40, respectively. It is seen that $E[Q]$ increases as $\mu_{1}$ increases but it decreases as $\lambda_{1}$ increases. Note that these numerical results may intuitively be understood as follows: The number of rented bikes increases as $\lambda_{1}$ increases, thus $E[Q]$ decreases; while the number of returned bikes increases as $\mu_{1}$ increases, this shows that $E[Q]$ increases as $\mu_{1}$ increases.

\section{Example two: Analysis of $E[N]$}

The left of figure 6 shows how the maximal stationary average number $E[N]$ of waiting 

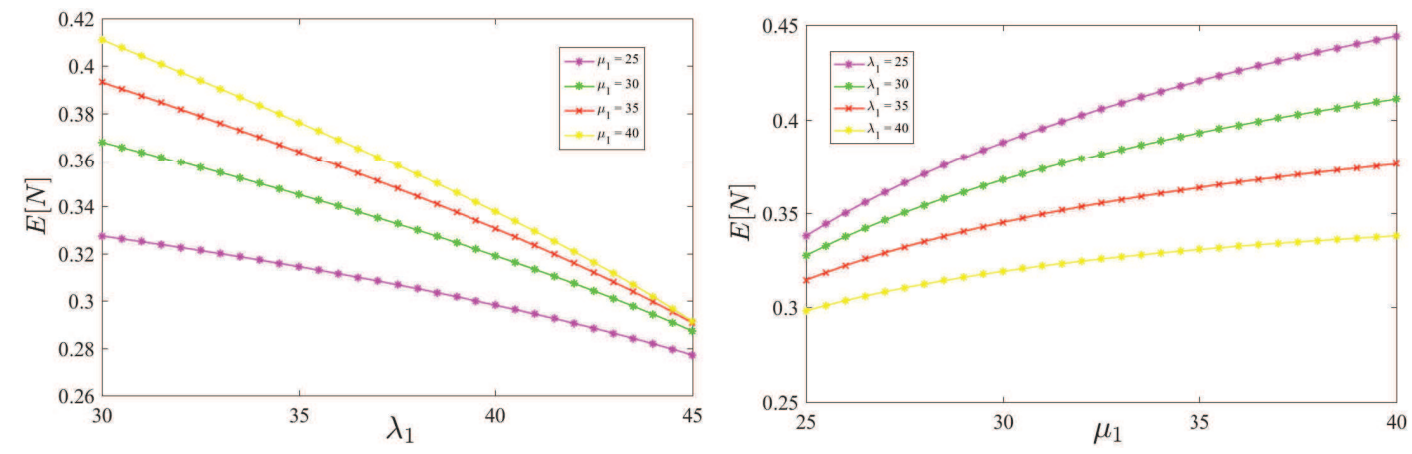

Figure 6: $E[N]$ vs. $\lambda_{1}$ and $\mu_{1}$

customers at the tagged station depends on $\lambda_{1} \in(30,45)$ when $\mu_{1}=25,30,35$ and 40, respectively. It is seen that $E[N]$ decreases as $\lambda_{1}$ increases but it increases as $\mu_{1}$ increases. The right of figure 6 shows how $E[N]$ depends on $\mu_{1} \in(25,40)$ when $\lambda_{1}=25,30,35$ and 40, respectively. It is seen that $E[N]$ increases as $\mu_{1}$ increases but it decreases as $\lambda_{1}$ increases. Note that the number of rented bikes increases as $\lambda_{1}$ increases, thus $E\left[N_{1}\right]$ increases but $E\left[N_{2}\right]$ decreases. On the other hand, the number of returned bikes increases as $\mu_{1}$ increases, so $E\left[N_{2}\right]$ increases but $E\left[N_{1}\right]$ decreases. Based on this, it is clear that $E\left[N_{2}\right]$ has more impact on $E[N]$ than $E\left[N_{1}\right]$ under the present parameter design.

\section{Example three: Analysis of the stationary weak-probability $\mathbf{p}_{w}$}

The left of Figure 7 shows how the stationary weak-probability $\mathbf{p}_{w}$ depends on $\lambda_{1} \in$ $(30,45)$ when $\mu_{1}=25,30,35$ and 40 , respectively. It is seen that $\mathbf{p}_{w}$ decreases as $\lambda_{1}$ increases but it increases as $\mu_{1}$ increases. The right of figure 7 shows how $\mathbf{p}_{w}$ depends on $\mu_{1} \in(25,40)$ when $\lambda_{1}=25,30,35$ and 40 , respectively. It is seen that $\mathbf{p}_{w}$ increases as $\mu_{1}$ increases but it decreases as $\lambda_{1}$ increases. Note that the number of rented bikes increases as $\lambda_{1}$ increases, thus $\sum_{k=-L}^{0} \pi_{k} e$ increases but $\sum_{k=K}^{K+L} \pi_{k} e$ decreases. On the other hand, the number of returned bikes increases as $\mu_{1}$ increases. This indicates that $\sum_{k=K}^{K+L} \pi_{k} e$ increases but $\sum_{k=-L}^{0} \pi_{k} e$ decreases. It is well understood that $\sum_{k=K}^{K+L} \pi_{k} e$ has more impact on $\mathbf{p}_{w}$ than $\sum_{k=-L}^{0} \pi_{k} e$ under the present parameter design.

\section{Example four: Analysis of the stationary strong-probability $\mathbf{p}_{s}$}

The left of figure 8 shows how the stationary strong-probability $\mathbf{p}_{s}$ depends on $\lambda_{1} \in$ $(30,45)$ when $\mu_{1}=25,30,35$ and 40 , respectively. It is seen that $\mathbf{p}_{s}$ decreases as $\lambda_{1}$ increases but it increases as $\mu_{1}$ increases. The right of figure 8 shows how $\mathbf{p}_{s}$ depends on $\mu_{1} \in(25,40)$ when $\lambda_{1}=25,30,35$ and 40 , respectively. It is seen that $\mathbf{p}_{s}$ increases 

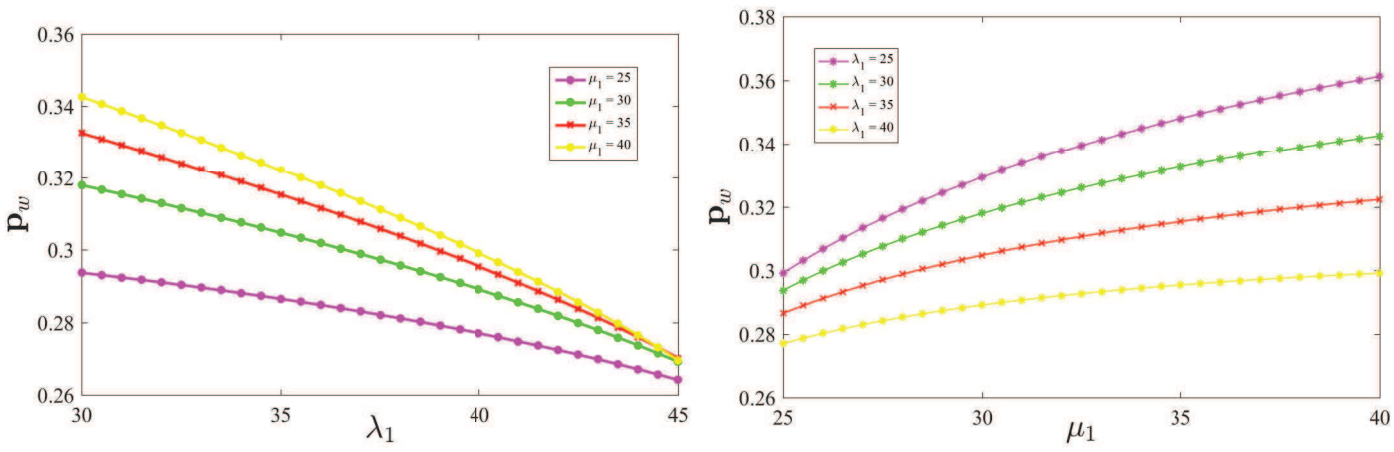

Figure 7: $\mathbf{p}_{w}$ vs. $\lambda_{1}$ and $\mu_{1}$
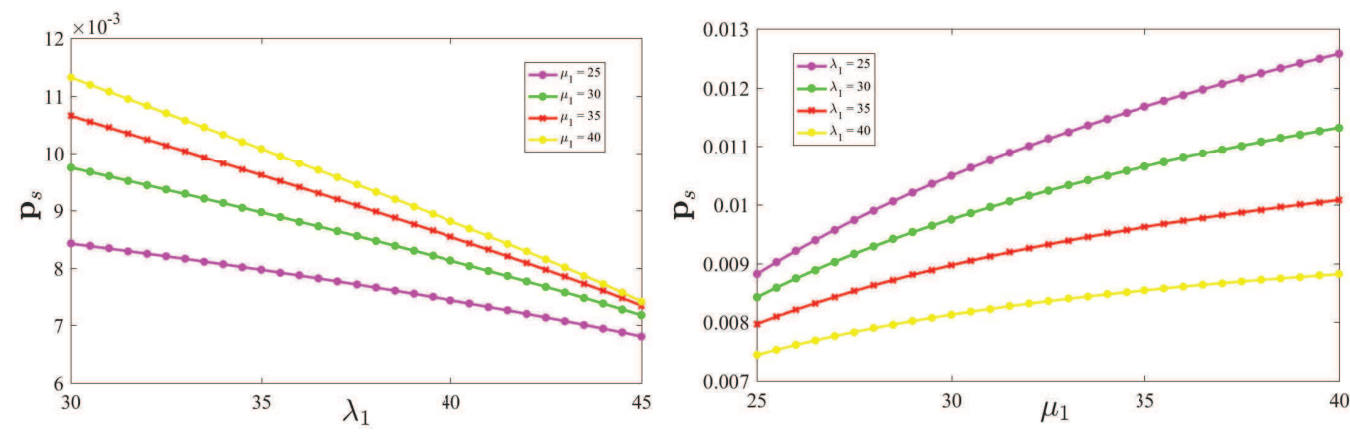

Figure 8: $\mathbf{p}_{s}$ vs. $\lambda_{1}$ and $\mu_{1}$

as $\mu_{1}$ increases but it decreases as $\lambda_{1}$ increases. Intuitively, the number of rented bikes increases as $\lambda_{1}$ increases, thus $\pi_{-L} e$ increases but $\pi_{K+L} e$ decreases. On the other hand, the number of returned bikes increases as $\mu_{1}$ increases, hence $\pi_{K+L} e$ increases but $\pi_{-L} e$ decreases. This demonstrates that $\pi_{K+L} e$ has more impact on $\mathbf{p}_{s}$ than $\pi_{-L} e$.

In the remainder of this section, we further observe some numerical impacts of the user's finite waiting rooms on system performance through the following two examples.

In Examples five and six, we take some common parameters as follows:

$$
K=20, C=5, m=2, \lambda_{2}=50, w=\left(\begin{array}{cc}
-1 & 1 \\
1 & -1
\end{array}\right)
$$

while the other parameters are conceretely chosen in each example for the target of specific observation.

Example five: Analysis of the stationary strong-probability $\mathbf{p}_{s}$ vs. $L$

In the left of Figure 9, We take $\lambda_{1}=45, \mu_{1}=\mu_{2}=20$ and $\beta=0.75$. The left of Figure 

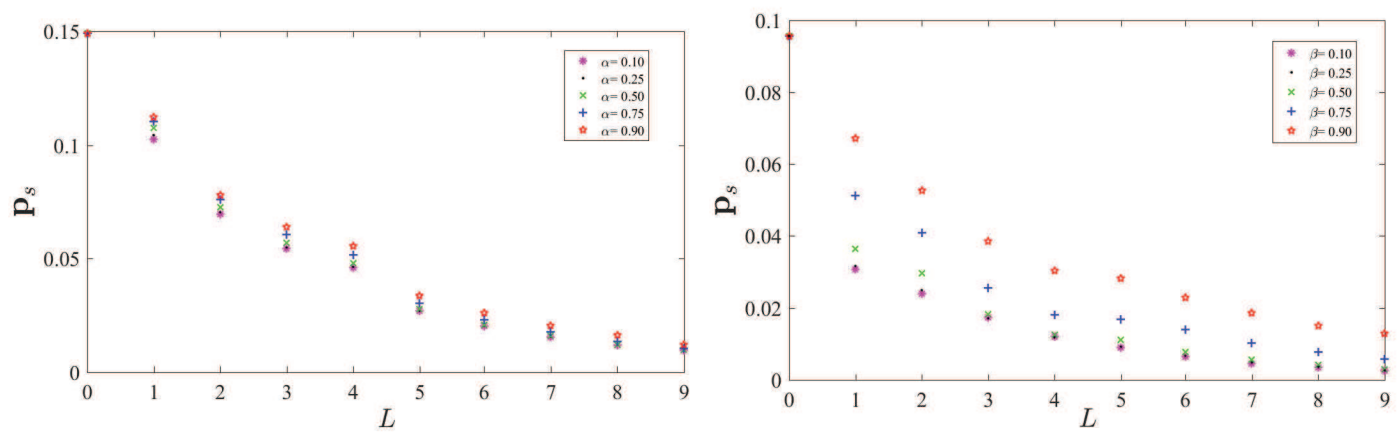

Figure 9: $\mathbf{p}_{s}$ vs. $L$

9 shows how the stationary strong-probability $\mathbf{p}_{s}$ depends on $L \in\{0,1,2,3,4,5,6,7,8,9\}$ when $\alpha=0.10,0,25,0.50,0.75,0.90$, respectively. It is seen that $\mathbf{p}_{s}$ decreases as $L$ increases but it increases as $\alpha$ increases.

In the right of Figure 9, we take $\lambda_{1}=55, \mu_{1}=\mu_{2}=10$ and $\alpha=0.75$. The right of Figure 9 shows how $\mathbf{p}_{s}$ depends on $L \in\{0,1,2,3,4,5,6,7,8,9\}$ when $\beta=$ $0.10,0,25,0.50,0.75,0.90$, respectively. It is seen that $\mathbf{p}_{s}$ decreases as $L$ increases but it increases as $\beta$ increases.

On the one hand, as $L$ increases, there are more and more waiting places provided for customers to wait for either an available bike (rent) or a vacant parking place (return), thus the probabilities of $\pi_{-L} e$ and $\pi_{K+L} e$ will decrease. This illustrates that $\mathbf{p}_{s}$ decreases. On the other hand, as $\alpha$ increases, more and more customers would like to wait for an available bike, thus $\pi_{-L} e$ increases but $\pi_{K+L} e$ decreases. As $\beta$ increases, more and more customers would like to wait for a vacant parking place, thus $\pi_{K+L} e$ increases but $\pi_{-L} e$ decreases.

Example six: Analysis of the ratio $v$ vs. $L$

In the left of Figure 10, we take $\lambda_{1}=45, \mu_{1}=\mu_{2}=20$ and $\beta=0.75$. The left of Figure 10 shows how the ratio $v$ depends on $L \in\{0,1,2,3,4,5,6,7,8,9\}$ when $\alpha=$ $0.10,0,25,0.50,0.75,0.90$, respectively. It is seen that the ratio $v$ decreases as $L$ increases but it increases as $\alpha$ increases.

In the right of Figure 10, we take $\lambda_{1}=55, \mu_{1}=\mu_{2}=10$ and $\alpha=0.75$. The right of Figure 10 shows how the ratio $v$ depends on $L \in\{0,1,2,3,4,5,6,7,8,9\}$ when $\beta=0.10,0,25,0.50,0.75,0.90$, respectively. It is seen that the ratio $v$ decreases as $L$ increases but it increases as $\beta$ increases. 

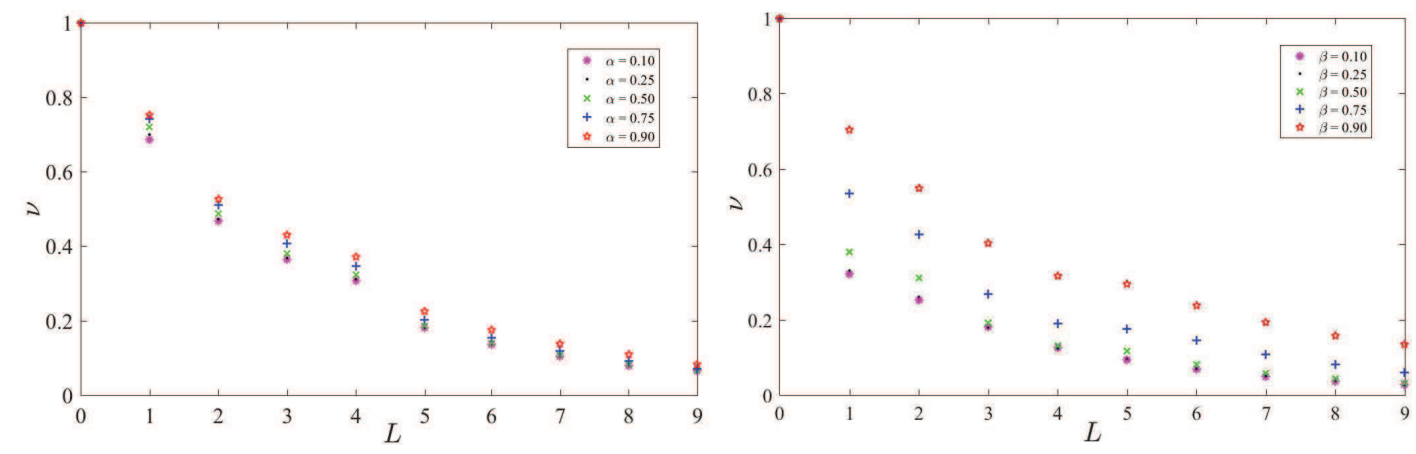

Figure 10: $\mathbf{p}_{s}$ vs. $v$

\section{Concluding Remarks}

In this paper, we describe a large-scale bike sharing system under Markovian environment, and develop a mean-field matrix-analytic method by combining the mean-field theory with the time-inhomogeneous queues as well as the nonlinear QBD processes. Furthermore, we apply the martingale limit theory to prove the asymptotic independence (or propagation of chaos) of this bike sharing system, and also study the limiting interchangeability as $N \rightarrow \infty$ and $t \rightarrow+\infty$. Based on this, we discuss the fixed point by means of a nonlinear QBD process so that we can give performance analysis of this bike sharing system. Notice that the mean-field matrix-analytic method is effective and efficient for, such as, designing reasonable architecture of a bike sharing system, finding a better path scheduling, improving inventory management, redistributing the bikes among stations or clusters in terms of truck scheduling, price optimization, application of intelligent information technologies and so forth.

This paper provides a clear way for how to use the mean-field matrix-analytic method to analyze performance measures of more general bike sharing systems in practice through three key parts: (1) Setting up a mean-field system of mean-field equations, (2) proving the asymptotic independence, and (3) analyzing performance measures of this bike sharing system by means of the fixed point. Therefore, the methodology and results of this paper give some new highlight on understanding performance measures and operations management of bike sharing systems. Along such a line, there are a number of interesting directions for potential future research, for example:

- Analyzing the fixed point for more general bike sharing systems in practice, and 
provide effective algorithms to deal with the nonlinear QBD processes;

- studying non-exponential riding-bike times and non-Poisson customer arrivals in bike sharing systems;

- introducing some better operations management, such as, redistribution of bikes by trucks, inventory management, applications of intelligent information techniques; and

- discussing large-scale bike sharing systems with different clusters or/and under price optimization.

\section{Acknowledgements}

Q.L. Li was supported by the National Natural Science Foundation of China under grant No. 71471160 and No. 71671158, and Natural Science Foundation of Hebei Province in China under grant No. G2017203277.

\section{References}

[1] Adelman D (2007) Price-directed control of a closed logistics queueing network. Operations Research 55(6): 1022-1038

[2] Benaim M, Le Boudec JY (2008) A class of mean-field interaction models for computer and communication systems. Performance Evaluation 65(11-12): 823-838

[3] Chen MF (2004) From Markov chains to non-equilibrium particle systems. World Scientific

[4] Darling RWR, Norris JR (2005) Structure of large random hypergraphs. The Annals of Applied Probability 15(1): 125-152

[5] Darling RWR, Norris JR (2008) Differential equation approximations for markov chains. Probability Surveys 5(1): 37-79

[6] DeMaio P (2009) Bike-sharing: history, impacts, models of provision, and future. Journal of Public Transportation 12(4): 41-56 
[7] Fishman E, Washington S, Haworth N (2013) Bike share: a synthesis of the literature. Transport Reviews 33(2): 148-165

[8] Fricker C, Gast N (2016) Incentives and regulations in bike-sharing systems with stations of finite capacity. EURO Journal on Transportation and Logistics 5(3): 261291

[9] Fricker C, Gast N, Mohamed A (2012) Mean field analysis for inhomogeneous bikesharing systems. In: International Meeting on Probabilistic, Combinatorial and Asymptotic Methods for the Analysis of Algorithms, pp 365-376

[10] Fricker C, Servel N (2016). Two-choice regulation in heterogeneous closed networks. Queueing Systems 82(1-2): 173-197

[11] Fricker C, Tibi D (2017) Equivalence of ensembles for large vehicle-sharing models. Ann. Appl. Probab 27(2): 883-916

[12] Gast N, Gaujal B (2010) A mean field model of work stealing in large-scale systems. ACM SIGMETRICS Performance Evaluation Review 38(1): 13-24

[13] Gast N, Gaujal B (2011) A mean field approach for optimization in discrete time. Discrete Event Dynamic Systems 21(1): 63-101

[14] Graham C (2000) Chaoticity on path space for a queueing network with selection of the shortest queue among several. Journal of Applied Probabability 37(1): 198-201

[15] Graham C (2005) Functional central limit theorems for a large network in which customers join the shortest of several queues. Probability Theory and Related Fields 131(1): $97-120$

[16] George DK, Xia CH (2011) Fleet-sizing and service availability for a vehicle rental system via closed queueing networks. European Journal of Operational Research 211(1): 198-207

[17] Kaspi M, Raviv T, Tzur M (2011) bike-sharing systems: user dissatisfaction in the presence of unusable bicycles. IISE Transactions 49(2): 144-158

[18] Labadi K, Benarbia T, Barbot JP, Hamaci S, Omari A (2015) Stochastic Petri Net modeling,simulation and analysis of public bicycle sharing systems. IEEE Transactions on Automation Scienceand Engineering 12(4): 1380-1395 
[19] Latouche G, Ramaswami V (1999) Introduction to matrix analytic methods in stochastic models. ASA-SIAM

[20] Leurent F (2012) Modelling a vehicle-sharing station as a dual waiting system: stochastic framework and stationary analysis. Available: HAL Id: hal-00757228

[21] Li QL (2010) Constructive computation in stochastic models with applications: The RG-factorizations. Springer and Tsinghua Press

[22] Li QL (2016) Nonlinear Markov processes in big networks. Special Matrices 4: 202217

[23] Li QL, Chen C, Fan RN, Xu L, Ma JY (2016) Queueing analysis of a large-scale bike sharing system through mean-field theory. Available: arXiv:1603.09560

[24] Li QL, Dai G, Lui JCS, Wang Y (2014) The mean-field computation in a supermarket model with server multiple vacations. Discrete Event Dynamic Systems 24(4): 473522

[25] Li QL, Du Y, Dai G, Wang M (2015) On a doubly dynamically controlled supermarket model with impatient customers. Computers \& Operations Research 55: 76-87

[26] Li QL, Fan RN, Ma JY (2016) A unified framework for analyzing closed queueing networks in bike sharing systems. In: Information Technologies and Mathematical Modelling: Queueing Theory and Applications. Springer, pp 177-191

[27] Li QL, Fan RN, Qian ZY (2017) A nonlinear solution to closed queueing networks for bike sharing systems with Markovian arrival processes and under an irreducible path graph. In: Queueing Theory and Network Applications, Lecture Notes in Computer Science, vol 10591. Springer, pp 118-140.

[28] Li QL, Lui JCS (2016) Block-structured supermarket models. Discrete Event Dynamic Systems 26(2): 147-182

[29] Li QL, Qian ZY, Fan RN (2017) Fluid and diffusion limits for bike sharing systems. In: Queueing Theory and Network Applications, Lecture Notes in Computer Science, vol 10591. Springer, pp 217-245

[30] Liggett TM (1985) Interacting particle systems. Springer 
[31] Martin JB, Suhov YM (1999) Fast Jackson networks. The Annals of Applied Probability 9(5): $854-870$

[32] Meddin R, DeMaio P (2012) The bike-sharing world map. URL http://www.metrobike.net

[33] Mitzenmacher MD (1996) The power of two choices in randomized load balancing. Ph.D. thesis, Department of Computer Science, University of California at Berkeley, USA

[34] Mukhopadhyay A, Karthik A, Mazumdar RR, Guillemin F (2017) Mean field and propagation of chaos in multi-class heterogeneous loss models. Performance Evaluation 91: 117-131.

[35] Neuts MF (1981) Matrix-geometric solutions in stochastic models: An algorithmic approach. The Johns Hopkins University Press: Baltimore

[36] Raviv T, Kolka O (2013) Optimal inventory management of a bike-sharing station. IIE Transactions 45(10): 1077-1093

[37] Raviv T, Tzur M, Forma I (2012) Static repositioning in a bike-sharing system: Models and solution approaches. Euro Journal of Transportation and Logistics 2(3): 187229

[38] Rogers LCG, Williams D (1994) Diffusions, Markov processes, and martingales, Vol. 1: foundations. John Wiley \& Sons: New York

[39] Rogers LCG, Williams D (2000) Diffusions, Markov processes and martingales, Vol. 2: Itô calculus. Cambridge University Press

[40] Shaheen S.A, Guzman SY, Zhang H (2010) Bike-sharing in Europe, the American and Asia: Past, present and future. Transportation Research Record: Journal of the Transportation Reaearch Board (2143): 159-167

[41] Schuijbroek J, Hampshire R, van Hoeve WJ (2013) Inventory rebalancing and vehicle routing in bike-sharing systems. Paper 1491, Tepper School of Business, Carnegie Mellon University 
[42] Shu J, Chou MC, Liu Q, Teo CP, Wang IL (2013) Models for effective deployment and redistribution of bicycles within public bicycle-sharing systems. Operations Research 61(6): 1346-1359

[43] Sznitman A (1989) Topics in propagation of chaos. In: Springer-Verlag Lecture Notes in Mathematics 1464, École d'Été de Probabilités de Saint-Flour XI, pp 165-251

[44] Turner SRE (1998) The effect of increasing routing choice on resource pooling. Probability in the Engineering and Informational Sciences 12(1): 109-124

[45] Vvedenskaya ND, Dobrushin RL, Karpelevich FI (1996) Queueing system with selection of the shortest of two queues: An asymptotic approach. Problems of Information Transmissions 32(1): 15-27

[46] Waserhole A, Jost V (2016) Pricing in vehicle sharing systems: Optimization in queuing networks with product forms. EURO Journal on Transportation and Logistics 5(3): $293-320$

[47] Waserhole A, Jost V, Brauner N (2013) Pricing techniques for self regulation in vehicle sharing systems. Electronic Notes in Discrete Mathematics 41: 149-156 